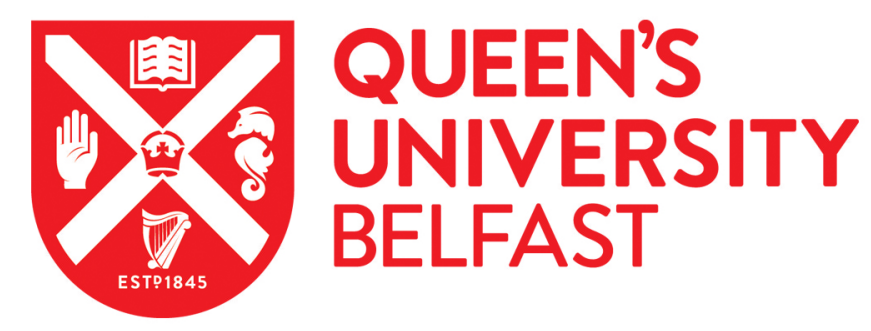

\title{
Stability Analysis and Implementation of a Decentralized Formation Control Strategy for Unmanned Vehicles
}

Yang, A., Naeem, W., Irwin, G. W., \& Li, K. (2014). Stability Analysis and Implementation of a Decentralized Formation Control Strategy for Unmanned Vehicles. IEEE Transactions on Control Systems Technology, 22(2), 706-720. https://doi.org/10.1109/TCST.2013.2259168

Published in:

IEEE Transactions on Control Systems Technology

Document Version:

Peer reviewed version

Queen's University Belfast - Research Portal:

Link to publication record in Queen's University Belfast Research Portal

\section{Publisher rights}

(C) 2013 IEEE. Personal use of this material is permitted. Permission from IEEE must be obtained for all other users, including reprinting/ republishing this material for advertising or promotional purposes, creating new collective works for resale or redistribution to servers or lists, or reuse of any copyrighted components of this work in other works. The final published version can be found here:

http://ieeexplore.ieee.org/xpl/articleDetails.jsp?arnumber=6517247

\section{General rights}

Copyright for the publications made accessible via the Queen's University Belfast Research Portal is retained by the author(s) and / or other copyright owners and it is a condition of accessing these publications that users recognise and abide by the legal requirements associated with these rights.

Take down policy

The Research Portal is Queen's institutional repository that provides access to Queen's research output. Every effort has been made to ensure that content in the Research Portal does not infringe any person's rights, or applicable UK laws. If you discover content in the Research Portal that you believe breaches copyright or violates any law, please contact openaccess@qub.ac.uk. 


\title{
Stability Analysis and Implementation of a Decentralized Formation Control Strategy for Unmanned Vehicles
}

\author{
Aolei Yang, Wasif Naeem, George W. Irwin, Fellow, IEEE, and Kang Li, Senior Member, IEEE
}

\begin{abstract}
This paper presents a new methodology for solving the multivehicle formation control problem. It employs a unique extension-decomposition-aggregation scheme to transform the overall complex formation control problem into a group of subproblems, which work via boundary interactions or disturbances. Thus, it is proved that the overall formation system is exponentially stable in the sense of Lyapunov, if all the individual augmented subsystems (IASs) are stable. Linear matrix inequality-based $H_{\infty}$ control methodology is employed to design the decentralized formation controllers to reject the impact of the formation changes being treated as boundary disturbances and guarantee the stability of all the IASs, consequently maintaining the stability of the overall formation system. Simulation studies are performed to verify the stability, performance, and effectiveness of the proposed strategy.
\end{abstract}

Index Terms-Decentralized formation control, formation stability, $H_{\infty}$ robust control, linear matrix inequality (LMI) optimization, Lyapunov stability.

\section{INTRODUCTION}

M ULTIVEHICLE formation control is one of the most important research areas in the field of cooperative control. Formation control is to control displacement and/or attitude of each vehicle within some workspace to accomplish a common task. Applications can be found in the fields of multirobots motion, air traffic control, collaborative mapping and exploration, unmanned aerial vehicle (UAV) formation patrol, autonomous underwater vehicle (AUV) formation cruise, and space satellite clustering [1]. It can be argued that a group of vehicles can perform tasks faster and more efficiently compared with a single vehicle. For example, a group of robots can be employed to move a large object, which may be difficult or impossible for a single robot; multiple UAVs and AUVs formation can achieve better area coverage for reconnaissance thereby improving mission success [2]; space satellite clustering can help to reduce the fuel consumption for propulsion and expands sensing capabilities.

Manuscript received May 3, 2012; revised February 14, 2013; accepted April 4, 2013. Manuscript received in final form April 17, 2013. This work was supported in part by the EPSRC under U.K.-China Science Bridge under Grant EP/G042594/1, and the China Scholarship Council. Recommended by Associate Editor H. Gao.

A. Yang is with the School of Mechatronic Engineering and Automation, Shanghai University, Shanghai 200444, China (e-mail: ayang02@qub.ac.uk).

W. Naeem, G. W. Irwin, and K. Li are with the School of Electronics, Electrical Engineering and Computer Science, Queen's University Belfast, Belfast BT9 5AH, U.K. (e-mail: w.naeem@qub.ac.uk; g.irwin@qub.ac.uk; k.li@qub.ac.uk).

Color versions of one or more of the figures in this paper are available online at http://ieeexplore.iee.org.

Digital Object Identifier 10.1109/TCST.2013.2259168
In the literature, there are four typically formation control strategies of importance: the behavior-based strategy, the leader-following approach, the virtual structure method, and the artificial potential approach. In this paper, a new decentralized formation control strategy is presented. The proposed scheme utilizes useful various characteristics from each of the above categories to develop a generalized cooperative control methodology. A unique process called extensiondecomposition-aggregation (EDA) is adopted to simplify the complex formation control problem. It is shown theoretically that the overall formation system is exponentially stable in the sense of Lyapunov. A review of the most widely used formation control strategies is now discussed. This will be followed by consideration of the approaches for formation stability analysis. The major contributions of this paper will finally be highlighted.

\section{A. Formation Control Strategies}

The behavior-based approach comes from the study of animal behaviors. It is decentralized and the control action of each element (node) in the formation is a weighted average of the control for each behavior. In [3], the author presented a standard behavior-based technique, which consists of several behaviors including maintain-formation, avoid-static-obstacle, avoid-robot, and move-to-goal. [4] also presented several behavioral strategies for the formation maneuver of a group of mobile robots as well as defining formation patterns to achieve a sequence of maneuvers. [5] used nonlinear attractor dynamics to design a dynamic control architecture. The behavior of each robot is generated as a time series of asymptotically stable states, which then contributes to the asymptotic stability of the overall formation control system. For this approach, its main advantage is that the collision avoidance problem can be easily dealt with due to the existing reactions between vehicles. However, the whole system is more complex and difficult to be analyzed mathematically.

In the leader-following approach, the leader vehicle maintains the given trajectory while the followers track a fixed relative distance from the designated neighboring vehicles. A classical leader-following approach was presented in [6], and a similar strategy was also applied in [7] for implementing the separation-bearing control and separation-separation control. Another studies and applications can be found in [8], and several control techniques, such as model predictive control [9] and sliding mode control [10], were employed to implement 
the leader-following strategy. The leader-following approach is decentralized, conceptually simple and widely employed in the multivehicle formation control. Its major drawback is that the leader becomes a single point of failure, whereas the followers usually demonstrate poor responses due to error propagation.

The virtual structure approach is commonly centralized and describes the whole formation as a single rigid body. Each vehicle has its own relative position in the body and tracks the desired trajectory, which is translated from the desired motion of the rigid body. The concept of a virtual structure was first introduced in [11], where the proposed algorithm iteratively fits the virtual structure to the robots positions, displaces the virtual structure in some desired directions and updates the robots positions. In other literatures, this approach were used in the formation control of spacecraft [12] and marine vehicles [13]. Moreover, in [14], decentralized formation control strategies via the virtual structure were introduced, which are more appropriate when a large number of spacecraft are involved. The strength of this approach is that all elements in the formation hold the same transient, and a tight formation during maneuver can then be implemented and maintained. However, collision avoidance is not possible due to the absence of interaction between the vehicles, as well as lack of explicit feedback to the formation.

The standard artificial potential field approach is based on applying the negative gradient of artificial potential function (APF) as control inputs to drive the overall formation to convergence. A framework for decentralized control of selforganizing swarm systems based on the APFs was presented in [15] and [16] employed social potential fields to a scalable multirobot formation. Moreover, in [17], the controller development was based on a new general potential function embedding a proposed $p$-times differential function. When the desired formation shape is achieved, the minimum value of the potential function will be attained, whereas it will be equal to infinity when a collision happens. This approach is decentralized, reactive, and can be beneficially combined with the behavior-based approach to make use of its benefits. However, it suffers from a problem with local minima and there is computational difficulty in designing a feasible APF.

In addition to the above four types of typical strategies, there are a number of other novel approaches. For instance, in [18], the notion of passivity evolved from network theory was used as a design tool to solve the group coordination problem. [19] employed decentralized overlapping feedback law for the formation of UAV to robustly stabilize the perturbed nominal dynamics of the subsystem. [20] used nonlinear contraction theory to study synchronization, which is related to the cooperative consensus problem.

Given the advantages and weaknesses of the approaches discussed above, an improved method for formation control commonly includes some, or all, of the following characteristics. First, it should be better to be a decentralized formation control architecture for avoiding the single point of failure, with all the vehicles having their own controllers and working jointly to maintain the stability of the overall formation. Second, in spite of no way to completely avoid the formation error propagation under the decentralized control architecture, the formation controller designed based on the proposed strategy should have the better performance to reject the error propagation than others. Third, the feasibility and performance of the overall formation should not be enormously degraded with an increase in the number of vehicles in the formation. Fourth, feedback to the formation and a reactive scheme between the vehicles should be included for dealing with inner and outer collisions. Fifth, not only should the formation maintenance be easy to express and analyze, but also the dynamics of any formation pattern change should be convenient to implement. Finally, the formation controller should be easily designed and computational efficient, which is of great significance for practical engineering applications.

\section{B. Approaches of Stability Analysis}

In addition to the above characteristics, the close-loop formation control methodology must be guaranteed to be stable, especially where there is a potential of loss of human life and property. Most research papers in this area make use of either algebraic graph theory or Lyapunov stability theory.

The use of graph theory in the analysis of interconnected systems is not new [21], and the current interest is in applying graph-theoretic concepts to the multivehicle formation problem. An application of graph theory was discussed in [22], where a directed graph was used to represent the communication network and to relate its topology with formation stability. In another literature, [23] presented a framework for describing the behaviors of robots in a formation, representing possible control graphs and the coordination of transitions with formation changes from one geometry to another.

In contrast, the Lyapunov stability theory is an alternative way to analyze the overall formation stability. In [24], a formation Lyapunov stability function is defined as a weighted sum of the control Lyapunov function for each vehicle to support the formation stability analysis. In [25], the idea of relative-position-based formation stability was proposed and the Lyapunov method was also used to design the decentralized controllers, along with an extended linear matrix inequality (LMI) to analyze the conditions required for formation stability. Moreover, paper [26] proposed a Lyapunovbased approach to give a sufficient condition to make all the agents converge to a common value, and a common Lyapunov function was explicitly constructed in the case of switching jointly connected topologies. In [27], a combination of the above two strategies was employed, where a few concepts from graph theory were borrowed to evaluate the controllability and observability of the individual system and a vector Lyapunov method was then applied to prove the stability of the multivehicle formation.

\section{Contributions of This Paper}

The purpose of this paper is to present a new methodology for multivehicle formation control and to analyze its associated formation stability. Although differing from the previous strategies, it necessarily builds on them and has the desired characteristics. In this approach, the EDA process is adopted to support the decentralized formation controller 
design and formation stability analysis. In the extension process, a virtual additional system (VAS) is employed to build the relationship between isolated vehicles and to produce an overall formation control system involving formation information. Decomposition of the overall formation system is then carried out to translate this complex formation system into a group of feasible subsystems interacting via boundary conditions. Here, such decomposed subsystems are referred to as individual augmented subsystems (IASs). A scalar Lyapunov function is next selected as an index for representing the stability of each IAS. These indices are finally aggregated to mathematically analyze the subsystem interactions and the stability of the overall formation using Lyapunov stability theory. It is concluded that the overall formation is exponentially stable in the sense of Lyapunov if each individual IAS in the formation is stable.

The nonlinear interaction or impact between the decomposed IASs is a reflection or indication of the local-formation error, and can be considered as an energy-bounded exogenous disturbance to a related IAS. A decentralized $H_{\infty}$ formation controller based on LMI is employed to stabilize each IAS and reject the disturbance. Using this idea, the complexity of the decentralized formation controller design can be reduced significantly. Results from a number of simulation studies, involving formation maintenance, change, and turning maneuver of vehicles, demonstrate the formation stability and the effectiveness of the proposed methodology.

The remainder of the paper is organized as follows. Section II outlines the problem formulation and preliminaries. Section III presents the new formation control methodology and the relevant mathematical stability analysis of the overall formation control system, while Section IV describes the decentralized formation controller design to a group of robots. Extensive simulation results are presented in Section $\mathrm{V}$ to illustrate the feasibility and verify the formation stability. The paper finally concludes with some discussion and suggestions for future work.

\section{Problem Formulation AND PREliminaries}

To implement the formation control, its control architecture and communication topology should be initially considered. Control architecture could either be centralized or decentralized. Here, a decentralized formation control strategy is adopted, which is more flexible, reliable and robust than the former. The communication topological structures, such as star, mesh, chain, and ring topology, represent the type of communication, which describes the relationship between the vehicles. Depending on the general requirement of minimal communication flow, a chain topology presented in [28] is employed here for sharing the mutual information within each local formation. With the decentralized control architecture and the chain communication topology, the definitions of formation and formation stability are mathematically presented in the following.

\section{A. Formation Definition}

To mathematically define the formation, a reference vehicle $(\mathrm{RV})$ can be selected by consensus between vehicles to

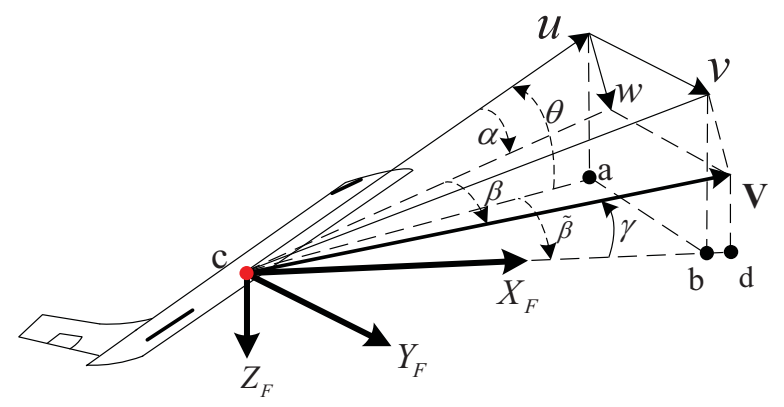

Fig. 1. VFRF of an aircraft.

determine the success of a formation maneuvering mission. An additional benefit of using the RV is to define the local formation coordinate system (LFCS). Note that the RV is different from the leader vehicle in the leader-following approach, and any vehicle within the formation can be chosen as the RV.

Before giving the definition of the LFCS, a new axes frame called as vehicle-carried formation reference frame (VFRF) is first defined. For vehicle's motion in 3-D space, the motional direction can be in common resolved into three orthogonal directions: horizontal forward, lateral, and vertical directions. Compared with the inertial reference frame $\left(X_{I}, Y_{I}, Z_{I}\right)$, the body-fixed reference frame $\left(X_{B}, Y_{B}, Z_{B}\right)$, stability reference frame $\left(X_{S}, Y_{S}, Z_{S}\right)$, and wind reference frame $\left(X_{W}, Y_{W}, Z_{W}\right)$, the VFRF denoted by $\left(X_{F}, Y_{F}, Z_{F}\right)$ axes is shown in Fig. 1, where an aircraft is given to illustrate the definition of the VFRF. Its origin lies on the center of gravity (CG) of the aircraft. The positive $X_{F}$ points forward and aligns to the level projection of the aircraft velocity vector $\left(\mathbf{V}=[u, v, w]^{T}\right)$. The positive $Z_{F}$ points toward the ground, and the positive $Y_{F}$ can be conveniently obtained by using the right-handed rule. Note that the VFRF is similar to the wind axes frame, but with a rotation angle $\gamma$ (flight-path angle) about the $Y_{W}$ or $Y_{F}$. Since the direction of the aircraft velocity vector keeps changing during flight, the $\left(X_{F}, Y_{F}, Z_{F}\right)$ axes system, unlike the body axes system, is not fixed. However, when an aircraft is flying in a level plane, it means that $\gamma=0 \mathrm{rad}$ and the VFRF is then consistent with the wind axes frame. Further, when the aircraft is under the situation of straight and level flight, it means that $\gamma=0 \mathrm{rad}$ and side-slip angle $\beta=0 \mathrm{rad}$, and consequently the VFRF, wind axes, and stability axes frames are consistent with one another.

With the definition of the VFRF, the LFCS can be defined conveniently, and provides a convenient way to describe the formation pattern in 3-D, a simple illustration being shown in Fig. 2, where vehicle 2 is selected as the RV within the group, and the LFCS denoted by $\left(X_{L}, Y_{L}, Z_{L}\right)$ always aligns to the RVs $\left(X_{F}, Y_{F}, Z_{F}\right)$ axes. In other words, the origin of the LFCS lies in the CG of the RV, and $X_{L}, Y_{L}$, and $Z_{L}$ being separately consistent with $X_{F}, Y_{F}$, and $Z_{F}$ axes of the RV. This implies that the LFCS axes are not fixed and always vary with the RVs position and direction due to the change of RVs attitude. The position vector of the $i$ th vehicle in the LFCS is defined as $\mathbf{p}_{\mathrm{Li}}=\left[p_{\mathrm{Lix}}, p_{\mathrm{Liy}}, p_{\mathrm{Liz}}\right]$, and the relative position between any two vehicles ( $i$ th and $j$ th) is given by $\mathbf{r}_{\mathrm{Lij}}=$ $-\mathbf{r}_{\mathrm{Lji}}=\mathbf{p}_{\mathrm{Li}}-\mathbf{p}_{\mathrm{Lj}}=\left[p_{\mathrm{Lix}}, p_{\mathrm{Liy}}, p_{\mathrm{Liz}}\right]-\left[p_{\mathrm{Ljx}}, p_{\mathrm{Ljy}}, p_{\mathrm{Ljz}}\right]$, 


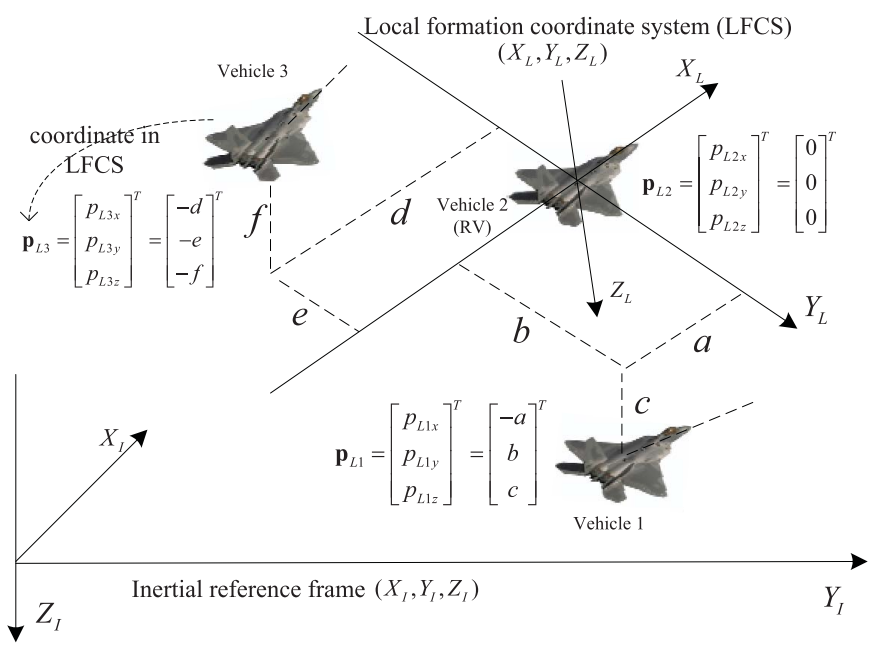

Fig. 2. Inertial reference frame and LFCS.

where $i, j=1,2, \ldots, N, i \neq j, L$ represents the LFCS and $N$ is the number of vehicles in the formation. Although the definitions of the VFRF and LFCS are based on the aircraft motion in 3-D space, this idea can be conveniently borrowed to deal with the multirobot formation maneuvering in 2-D space.

It is essential to note that only using the relative distance between the vehicles is not sufficient to define a formation, since the motion of each vehicle in 3-D space has six dynamic degrees of freedom defining the position and attitude, and unstable attitude will definitely affect the above relative distance. On the other hand, it is believed that when a formation change is needed, the related attitude should generally be regulated by a formation control algorithm until all the vehicles achieve their new desired relative distances. Thus, a complete formation definition in Euclidean space should consist of the relative distances and states of all the vehicles, which is expressed by (1) and (2)

$$
\begin{aligned}
F_{\mathrm{rd}} & \triangleq\left\{\mathbf{r}_{L i j}: i, j=1,2, \ldots, N ; i \neq j\right\} \\
\tilde{\mathbf{x}} & =\left[\tilde{\mathbf{x}}_{1}, \tilde{\mathbf{x}}_{2}, \ldots, \tilde{\mathbf{x}}_{i}, \ldots, \tilde{\mathbf{x}}_{N}\right]^{T}
\end{aligned}
$$

where $F_{\mathrm{rd}}$ is the global formation set including all the relative distances, $\tilde{\mathbf{x}}_{i}$ is the state vector of the $i$ th vehicle, and $\tilde{\mathbf{x}}$ is the combination of all the vehicles' state vectors within the formation.

\section{B. Formation Stability Definition}

1) Formation Stability Definition 1: The formation with $N$ vehicles is asymptotically stable in the sense of Lyapunov if both (3) and (4) are satisfied

$$
\begin{array}{r}
\lim _{t \rightarrow+\infty}\left\|F_{\mathrm{rd}}-F_{e}\right\|=0 \\
\lim _{t \rightarrow+\infty}\left\|\tilde{\mathbf{x}}-\tilde{\mathbf{x}}_{e}\right\|=0
\end{array}
$$

where $t$ is the elapsed time, $F_{e}$ is the equilibrium shape of the overall formation, $\tilde{\mathbf{x}}_{e}=\left[\tilde{\mathbf{x}}_{e_{-} 1}, \tilde{\mathbf{x}}_{e_{-} 2}, \ldots, \tilde{\mathbf{x}}_{e_{-} N}\right]^{T}$ consists of the equilibrium states of $\tilde{\mathbf{x}}_{i}, i=1,2, \ldots, N$. This formation stability definition means that both the states of all the vehicles and the relative distances between them should converge to steady states. Note that achieving the above equilibrium shape

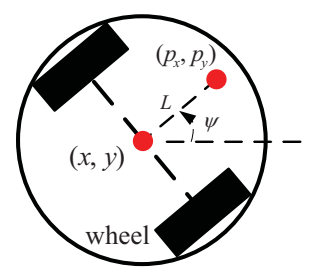

Fig. 3. Omnidirectional mobile robot.

and steady-state values is sufficient to ensure the stability of the overall formation. However, it does not necessarily mean that the achieved stable formation geometry is the same as the desired or reference formation shape $\left(F_{d}\right)$. As given in (5)

$$
\Delta F=F_{\mathrm{rd}}-F_{d}
$$

there generally exists a steady-state formation error $(\Delta F)$, which can in general be expressed by the difference between $F_{\text {rd }}$ and $F_{d}$. From the viewpoint of application, this error is undesired and needs to be eliminated by using additional compensators.

Furthermore, it is obvious that $F_{\text {rd }}$ in (1) and (3) shows a global expression of the overall formation. With the adopted decentralized control architecture and the chain topology, it is assumed that $F_{\text {rd }}$ can be decomposed into a set of local-formation representations, i.e., $F_{\text {rd }} \triangleq\left\{F_{\text {rd_1 } 1}, F_{\text {rd } \_2}, \ldots\right.$, $\left.F_{\text {rd } \_i}, \ldots, F_{\text {rd } \_N}\right\}$, where $F_{\text {rd } \_i}$ corresponds to the localformation representation of the $i$ th vehicle. Supposing that it can be expressed by a vector $\mathbf{x}_{F_{\text {rd } \_i}}$ to reflect the variation of the associated local formation, the state vector $\tilde{\mathbf{x}}_{i}$ of the $i$ th vehicle can then be augmented as

$$
\mathbf{x}_{i}=\left[\tilde{\mathbf{x}}_{i}, \mathbf{x}_{F_{\text {rd } \_i}}\right]^{T}
$$

where $\mathbf{x}_{i}$ is considered as the augmented state vector derived from the original one, $\tilde{\mathbf{x}}_{i}$ of the $i$ th vehicle. The Formation stability definition 1 is then redefined as definition 2 below.

2) Formation Stability Definition 2: The formation with $N$ vehicles is asymptotically stable in the sense of Lyapunov if (7) is satisfied

$$
\lim _{t \rightarrow+\infty}\left\|\mathbf{X}-\mathbf{X}_{e}\right\|=0
$$

where $\mathbf{X}=\left[\mathbf{x}_{1}, \mathbf{x}_{2}, \ldots, \mathbf{x}_{i}, \ldots, \mathbf{x}_{N}\right]^{T}$ consists of all the state vectors of the augmented systems derived from the original vehicle systems, and $\mathbf{X}_{e}=\left[\mathbf{x}_{e_{-} 1}, \mathbf{x}_{e_{-} 2}, \ldots, \mathbf{x}_{e_{-} i}, \ldots, \mathbf{x}_{e_{-} N}\right]^{T}$ is the equilibrium states including all the individual equilibrium states of the augmented systems. The above two formation stability definitions show the different forms, but are equivalent to each other.

\section{Modeling and Feedback Linearization of Vehicles}

A number of research papers, such as [23] and [29], employed a kinematic model in their designs. However, here, an omnidirectional mobile robot [4] shown in Fig. 3 is chosen, and the corresponding nonlinear dynamical model is given 

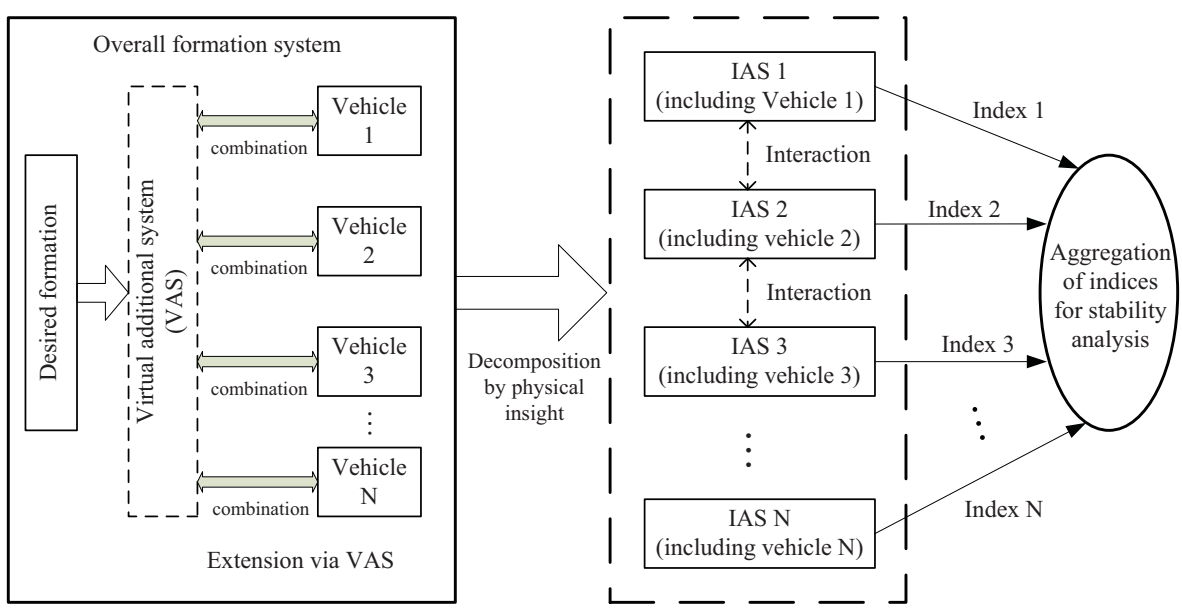

Fig. 4. Process of EDA.

by (8)

$$
\begin{aligned}
\left\{\begin{array}{l}
\dot{x}_{i}=v_{i} \cos \psi_{i} \\
\dot{y}_{i}=v_{i} \sin \psi_{i} \\
\dot{v}_{i}=F_{i} / M_{i} \\
\dot{\psi}_{i}=\omega_{i} \\
\dot{\omega}_{i}=\tau_{i} / J_{v i}
\end{array}\right. & \rightarrow\left[\begin{array}{c}
\dot{x}_{i} \\
\dot{y}_{i} \\
\dot{v}_{i} \\
\dot{\psi}_{i} \\
\dot{\omega}_{i}
\end{array}\right] \\
= & {\left[\begin{array}{c}
v_{i} \cos \psi_{i} \\
v_{i} \sin \psi_{i} \\
0 \\
\omega_{i} \\
0
\end{array}\right]+\left[\begin{array}{ll}
0 & 0 \\
0 & 0 \\
1 & 0 \\
0 & 0 \\
0 & 1
\end{array}\right]\left[\begin{array}{l}
F_{i} / M_{i} \\
\tau_{i} / J_{v i}
\end{array}\right] }
\end{aligned}
$$

where $i$ is the vehicle index, $\left(x_{i}, y_{i}\right)$ is the planar position, $\psi_{i}$ is the heading or yaw angle, $v_{i}, \omega_{i}$ are the translational and angular velocities, $M_{i}, I_{\mathrm{vi}}$ are the mass and inertial moment of the vehicle, $F_{i}, \tau_{i}$ are the applied forces and torques, and the inputs $\left[F_{i} / M_{i}, \tau_{i} / I_{\mathrm{vi}}\right]^{T}$ are the linear and angular accelerations. Because of the nonholonomic constraint ( $\dot{x}_{i} \sin \psi_{i}-\dot{y}_{i} \cos \psi_{i}=0$ ) to this model, the reference position of the $i$ th vehicle is defined as $\left(p_{\mathrm{xi}}, p_{\mathrm{yi}}\right)$

$$
\left\{\begin{array}{l}
p_{\mathrm{xi}}=x_{i}+L_{i} \cos \psi_{i} \\
p_{\mathrm{yi}}=y_{i}+L_{i} \sin \psi_{i}
\end{array}\right.
$$

Here, $L_{i}$ is the vertical distance between the reference point to the wheel axis. This model can be linearized using input-output feedback linearization to produce a 2-D doubleintegrator model given by (10) [4]

$$
\left[\begin{array}{c}
\ddot{p}_{\mathrm{xi}} \\
\ddot{p}_{\mathrm{yi}}
\end{array}\right]=\left[\begin{array}{cc}
\cos \psi_{i} & -\sin \psi_{i} \\
\sin \psi_{i} & \cos \psi_{i}
\end{array}\right]\left[\begin{array}{c}
-L_{i} \omega_{i}+F_{i} / M_{i} \\
v_{i} \omega_{i}+L_{i} \tau_{i} / I_{\mathrm{vi}}
\end{array}\right]=\left[\begin{array}{l}
u_{\mathrm{xi}} \\
u_{\mathrm{yi}}
\end{array}\right] \text {. }
$$

Let $\ddot{P} \triangleq\left[\ddot{p}_{\mathrm{xi}}, \ddot{p}_{\mathrm{yi}}\right]^{T}$ and $u_{i} \triangleq\left[u_{\mathrm{xi}}, u_{\mathrm{yi}}\right]^{T}$, then the resultant input-output dynamics of each nonlinear vehicle is represented by the double integrator, $\ddot{P}_{i}=u_{i}$, which will be used in the modeling of the IASs in the following.

\section{Formation Control Methodology}

Most formation control approaches are direct methods and are based on direct distance or position errors. Such methods are easy to understand, but are not necessarily convenient for analysis and implementation purpose. Here, the EDA scheme
[30] is described to provide a framework for the decentralized formation controller design and formation stability analysis.

\section{A. EDA Strategy}

It has long been recognized that a certain complex system made up of interacting elements can be decomposed into subsystems of lower dimensionality, thus simplifying the solution and analysis. Those individual subsystem solutions are then combined in some way to provide an overall system response. Motivated by this idea, the EDA strategy shown in Fig. 4 transforms the overall complex formation control system into a group of subproblems, which interact via boundary interactions.

In multivehicle formation control, each vehicle is a separate entity in formation space, i.e., there is generally no explicit relationship among the vehicles to represent their formation statuses. The strategy here is to introduce a VAS, which has three main functions: 1) to build a relationship between the isolated vehicles leading to a new overall formation system; 2) to involve the desired formation variables or parameters, which could be combined with the related individual vehicle model; and 3) to support the subsequent decomposition and simplify the stability analysis of the overall formation. Note that the VAS is merely an algorithm to act as an interaction bridge providing each vehicle with the capability of sensing its local-formation states, which can then be used for the formation controller design.

Further, because the overall formation system involving many variables is difficult to handle as a whole, it is natural to decompose it into several local-formation subsystems. However, it should be noted that there is no general systematic procedure for decomposing such a complex dynamical formation control system. Here, it is assumed that the overall formation system can be decomposed into $N$ individual subsystems, each being called as an IAS as its state vector combines the state vector, $\tilde{\mathbf{x}}_{i}$ of the original vehicle model, and the decomposed local-formation vector, $\mathbf{x}_{F_{\text {rd_i }}}$, which is shown in (6).

In light of the above definitions and descriptions, the procedure for analyzing the whole formation stability is as follows.

1) All the decomposed subsystems or IASs are used to design the decentralized formation controllers, the 
interactions between them being considered as the bounded exogenous disturbances, which reflect the changes in the formation.

2) The formation stability of each IAS, involving several state variables, is represented by a single variablethe Lyapunov function (say $V_{i}$ for $i$ th IAS), which is considered as an index for representing the localformation stability of each decomposed subsystem. This approach simplifies the stability problem, although it sacrifices detailed information about the state variables of each IAS [31].

3) If each IAS can be stabilized by a designed decentralized formation controller and the bounded disturbances can also be rejected, these indices $\left(V_{i}, i=1,2, \ldots, N\right)$ are then aggregated and the interactions between them are studied to mathematically analyze the stability of the overall formation.

Generally, the $N$ IASs are associated with the $N$ scalar Lyapunov functions $\left(V_{i}, i=1,2, \ldots, N\right)$, and each determines the stability property of an IAS. These scalar functions are considered as components of the overall Lyapunov function $\mathbf{V}$. A differential inequality is formed in terms of this function, using the original inequalities of the decomposed IASs. The stability of the overall formation control system can then be determined by considering only the differential inequalities involving the $N$ Lyapunov functions. This brings about a considerable reduction in the dimensionality of the formation stability problem.

With the above description, the determination of the VAS is the strategic point, because the stability of the overall formation is completely dependent on it. In other words, if the suitable VAS is found, mathematical stability analysis and implementation of the decentralized formation controller become feasible and convenient. In the following, coupled multiple inverted pendulums (CMIP) system is employed as a candidate VAS, and the relationship between the CMIP and the multivehicle formation system will be presented in detail.

\section{B. Relationship Between CMIP and Multivehicle Formation System}

The typical CMIP system is an extension of the two inverted pendulums (TIP) system discussed in [32]. The chained CMIP system shown in Fig. 5 displays a feasible formation topology structure, chain formation topology, which is used to illustrate the interaction between each subsystems. It should be noted that this employed chain topology is not a unique candidate to build the vehicles' interaction. For instance, star, mesh, tree, and ring topologies can be also applied to the CMIP system.

The mathematical modeling and analysis of the chained CMIP system is detailed in Appendix, where the achieved important result is that if all the individual pendulums are stable, the resultant torque of the whole CMIP system is equal to zero whatever the interconnection formation topology is. In other words, the chosen topology does not affect the property of the CMIP system. For the convenience of description, the chained CMIP system is chosen as the VAS in the following text.

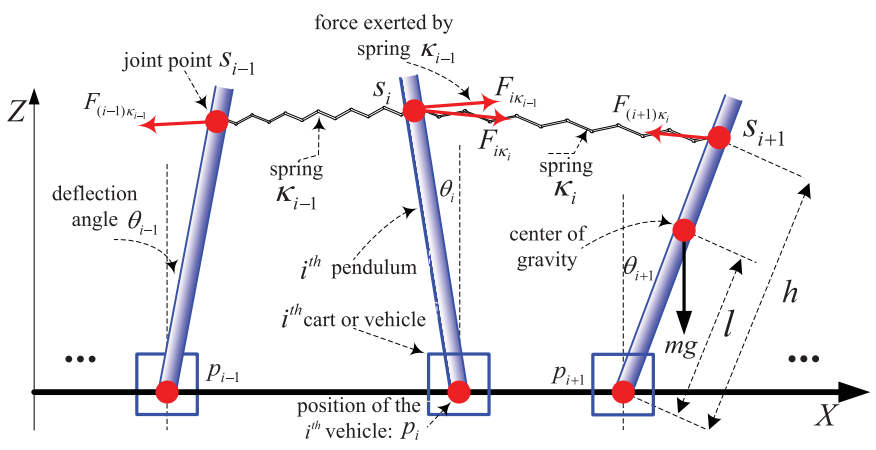

Fig. 5. CMIPs system.

The relationship between the CMIP and multivehicle formation system can be intuitively explained by analogy with the motion of each cart of the CMIP system. The interaction exerted by a virtual spring between two pendulums can be considered as the communication channel between two vehicles. Here, the magnitude of the torque from the spring force depends on the spring coefficient $\left(k_{S}\right)$, the free or natural length $\left(l_{\kappa_{i}}\right)$ and the relative distances between the vehicles. One approach of constructing the relationship is to consider the natural lengths of springs as the desired formation parameters or variables. If a formation is not stable, the force or torque exerted by the springs must then impact the pendulums and result in a change in their deflection angles $\theta_{i}$. This implies that the variation of $\theta_{i}$ is a reflection or indication of the formation error and it is referred to as a local-formation variable. Thus, the main aim of the decentralized formation controller design can be translated into the regulation problem of the localformation variable by maneuvering the formation vehicles. These will automatically cause the virtual springs to return to their balanced state, i.e., the overall formation becomes stable. Further, if all the springs return to their natural states or all the deflection angles are equal to zero, this in turn implies that the overall formation is stable with zero formation error.

Since the VAS or the CMIP is a virtual system, its parameters can be altered by the control algorithm according to the physical dynamics and engineering design requirements of the formation patterns. For example, if the natural lengths of the springs are dynamically redefined, the overall formation shape will likewise be changed. One of the most interesting aspects of this strategy is that it can be applied to handle the vehicle collision avoidance problem, which is of prime importance in the maneuvering of the multiple vehicles.

Recalling the EDA scheme shown in Fig. 4, the overall formation system based on the designed CMIP system is then partitioned or decomposed at the spring positions to generate a group of IASs, where each IAS is the combination of a real individual vehicle and a virtual inverted pendulum, which consists of the local-formation variable $\theta_{i}$. Based on the definition of the augmented state vector in (6), $\theta_{i}$ corresponds to $\mathbf{x}_{F_{\text {rd } \_i}}$ and the augmented state vector $\mathbf{x}_{i}$ is then the state vector of the $i$ th IAS, which will be used to analyze the overall formation stability.

Furthermore, since the interaction between the decomposed IASs reflect variations of the actual formation and there inevitably exist uncertainties in the modeling, it is usually 
difficult to precisely express and analyze such nonlinear effects on each IAS. From the perspective of the convenient implementation of decentralized formation control architecture, a novel idea for dealing with this is to consider the impact of such nonlinear interaction as energy-bounded exogenous disturbance to the relevant IAS. A number of techniques including robust control theory and active disturbance rejection control [33], are then employed to reject such interaction. This aspect is of great significance for practical application, since it greatly reduces the complexity of the decentralized controller design. Thus, the original complex formation control problem, typically involving complicated cooperative motions of many vehicles, is now redefined in terms of the stability of each IAS. The relationship between the stability of the overall formation and the stability of each IAS will be discussed in the next subsection.

\section{Formation Stability Analysis}

In general, it is obvious that ensuring the stability of all the individual vehicles in the formation is not sufficient to prove the stability of the overall formation. However, the ultimate goal of the present exposition is to show rigorously that the overall formation control system, when synthesized from the individually stable IASs, is mathematically stable.

Similar to the model of the CMIP given by (48), it is assumed that the overall formation control system denoted by $\mathbf{S}$, can be linearized and decomposed into $N$ coupled IASs, $\mathbf{S}_{i},(i=1,2, \ldots, N)$, as shown in

$$
\mathbf{S}:\left\{\begin{array}{l}
\mathbf{S}_{1}: \dot{\mathbf{x}}_{1}=A_{1} \mathbf{x}_{1}+h_{12}\left(t, \mathbf{x}_{2}\right) \\
\mathbf{S}_{2}: \dot{\mathbf{x}}_{2}=A_{2} \mathbf{x}_{2}+h_{21}\left(t, \mathbf{x}_{1}\right)+h_{23}\left(t, \mathbf{x}_{3}\right) \\
\vdots \\
\mathbf{S}_{i}: \dot{\mathbf{x}}_{i}=A_{i} \mathbf{x}_{i}+h_{i(i-1)}\left(t, \mathbf{x}_{i-1}\right)+h_{i(i+1)}\left(t, \mathbf{x}_{i+1}\right) \\
\vdots \\
\mathbf{S}_{N}: \dot{\mathbf{x}}_{N}=A_{N} \mathbf{x}_{N}+h_{N(N-1)}\left(t, \mathbf{x}_{N-1}\right)
\end{array}\right.
$$

where $\mathbf{x}_{i}$ is the state vector of the augmented system or the $i$ th IAS, $h_{i(i-1)}\left(t, \mathbf{x}_{i-1}\right)$ and $h_{i(i+1)}\left(t, \mathbf{x}_{i+1}\right)$ are derived from the connected springs and represent the interactions of the $i$ th IAS with its neighboring $(i-1)$ th and $(i+1)$ th IAS, respectively.

Now consider the stability of the corresponding decoupled linear subsystems $\tilde{\mathbf{S}}_{1}, \tilde{\mathbf{S}}_{2}, \ldots, \tilde{\mathbf{S}}_{N}$ by ignoring the above interactions

$$
\begin{aligned}
& \tilde{\mathbf{S}}_{1}: \dot{\mathbf{x}}_{1}=A_{1} \mathbf{x}_{1} ; \quad \tilde{\mathbf{S}}_{2}: \dot{\mathbf{x}}_{2}=A_{2} \mathbf{x}_{2} ; \ldots \\
& \tilde{\mathbf{S}}_{i}: \dot{\mathbf{x}}_{i}=A_{i} \mathbf{x}_{i} ; \quad \ldots ; \tilde{\mathbf{S}}_{N}: \dot{\mathbf{x}}_{N}=A_{N} \mathbf{x}_{N} .
\end{aligned}
$$

For the stability of $\tilde{\mathbf{S}}_{i}$ in (12), a scalar function $V_{i}\left(\mathbf{x}_{i}\right)$ is defined

$$
V_{i}\left(\mathbf{x}_{i}\right)=\left(\mathbf{x}_{i}^{T} P_{i} \mathbf{x}_{i}\right)^{1 / 2}, \quad(i=1,2, \ldots, N)
$$

as a candidate Lyapunov function. Thus, for any choice of the positive definite matrix $Q_{i}$, there exists a positive definite matrix $P_{i}$ as the solution to the Lyapunov matrix equation

$$
A_{i}^{T} P_{i}+P_{i} A_{i}=-Q_{i}
$$

The time derivative of the function $V_{i}\left(\mathbf{x}_{i}\right)$ along the solution of the subsystem $\tilde{\mathbf{S}}_{i}$ is therefore given by (15), [34]

$$
\dot{V}_{i}=\left(\operatorname{grad} V_{i}\right)^{T} \dot{\mathbf{x}}_{i}=\left(\operatorname{grad} V_{i}\right)^{T} A_{i} \mathbf{x}_{i}=-\frac{1}{2} V_{i}^{-1}\left(\mathbf{x}_{i}^{T} Q_{i} \mathbf{x}_{i}\right)
$$

where $\left(\operatorname{grad} V_{i}\right)=V_{i}^{-1} P_{i} \mathbf{x}_{i}^{T}$. From the above equations, the estimates for $V_{i}\left(\mathbf{x}_{i}\right), \dot{V}_{i}\left(\mathbf{x}_{i}\right)$ and $\left\|\operatorname{grad} V_{i}\right\|$ are given by [35]

$$
\left\{\begin{array}{l}
c_{i 1}\left\|\mathbf{x}_{i}\right\| \leq V_{i}\left(\mathbf{x}_{i}\right) \leq c_{i 2}\left\|\mathbf{x}_{i}\right\| \\
\dot{V}_{i}\left(\mathbf{x}_{i}\right) \leq-c_{i 3}\left\|\mathbf{x}_{i}\right\| \\
\left\|\operatorname{grad} V_{i}\right\| \leq c_{i 4}
\end{array} \quad(i=1,2, \ldots, N)\right.
$$

where

$$
\begin{aligned}
c_{i 1} & =\lambda_{m}^{1 / 2}\left(P_{i}\right), & c_{i 2} & =\lambda_{M}^{1 / 2}\left(P_{i}\right) \\
c_{i 3} & =\frac{1}{2} \frac{\lambda_{m}\left(Q_{i}\right)}{\lambda_{M}^{1 / 2}\left(P_{i}\right)}, & c_{i 4} & =\frac{\lambda_{M}\left(P_{i}\right)}{\lambda_{m}^{1 / 2}\left(P_{i}\right)}
\end{aligned}
$$

and the property of a positive semidefinite symmetric matrix $(M)$ is used, that is, $\lambda_{m}(M) \mathbf{x}^{T} \mathbf{x} \leq \mathbf{x}^{T} M \mathbf{x} \leq \lambda_{M}(M) \mathbf{x}^{T} \mathbf{x}$ and $\mathbf{x}^{T} \mathbf{x}=\|\mathbf{x}\|^{2}$, where $\lambda_{M}$ and $\lambda_{m}$ are the maximum and minimum eigenvalues of the indicated matrix, respectively. Note that the coefficients $c_{i 1}, c_{i 2}, c_{i 3}$, and $c_{i 4}$ are positive because of the positive definite property of the matrices $P_{i}$ and $Q_{i}$.

Now the Lyapunov function $V_{i}\left(\mathbf{x}_{i}\right)$ is used as the stability index for each linear subsystem $\tilde{\mathbf{S}}_{i}$. Furthermore, the derivative $\dot{V}_{i}$ along the solutions of the interacted IAS $\left(\mathbf{S}_{i}\right)$ in (11) is given by

$$
\begin{aligned}
\dot{V}_{i}= & \left(\operatorname{grad} V_{i}\right)^{T} \dot{\mathbf{x}}_{i}=\left(\operatorname{grad} V_{i}\right)^{T} \\
& \times\left[A_{i} \mathbf{x}_{i}+h_{i(i-1)}\left(t, \mathbf{x}_{i-1}\right)+h_{i(i+1)}\left(t, \mathbf{x}_{i+1}\right)\right] \\
= & \left(\operatorname{grad} V_{i}\right)^{T} A_{i} \mathbf{x}_{i}+\left(\operatorname{grad} V_{i}\right)^{T} \\
& \times\left[h_{i(i-1)}\left(t, \mathbf{x}_{i-1}\right)+h_{i(i+1)}\left(t, \mathbf{x}_{i+1}\right)\right] .
\end{aligned}
$$

Note that the term, $\left(\operatorname{grad} V_{i}\right)^{T} A_{i} \mathbf{x}_{i}$, is associated with the decoupled system (12), and the expression, $\left\|\mathbf{x}_{i}\right\| \geq c_{i 2}^{-1} V_{i}\left(\mathbf{x}_{i}\right)$, follows from the first inequality in (16). So, there exists an inequality given by

$$
\left(\operatorname{grad} V_{i}\right)^{T} A_{i} \mathbf{x}_{i} \leq-c_{i 3}\left\|\mathbf{x}_{i}\right\| \leq-c_{i 3} c_{i 2}^{-1} V_{i} .
$$

Theorem 1 (Overall Formation Stability): Let the virtual CMIP system be combined with all the isolated vehicles for generating an overall formation control system $\mathbf{S}$, and it is further decomposed into a group of subsystems or IASs, $\mathbf{S}_{i}, i=1,2, \ldots, N$, as modeled in (11). If all the IASs can be stabilized by using any approach, the overall formation system is not only asymptotically, but also exponentially stable in the sensor of Lyapunov within a neighborhood of the desired formation.

Proof: The stability of the overall formation system, S, can be represented by considering the interactions between the Lyapunov functions $V_{i}\left(\mathbf{x}_{i}\right),(i=1,2, \ldots, N)$ for the IASs. A function given in (20) defines the Lyapunov function $\mathbf{V}(\mathbf{X})$ for $\mathbf{S}$, which is the weighted sum of all the individual Lyapunov functions $V_{i}\left(\mathbf{x}_{i}\right)$

$\mathbf{V}(\mathbf{X})=\sum_{i=1}^{N} k_{i} V_{i}\left(\mathbf{x}_{i}\right)=k_{1} V_{1}\left(\mathbf{x}_{1}\right)+k_{2} V_{2}\left(\mathbf{x}_{2}\right)+\cdots+k_{N} V_{N}\left(\mathbf{x}_{N}\right)$ 
where $\mathbf{X}=\left[\mathbf{x}_{1}, \mathbf{x}_{2}, \ldots, \mathbf{x}_{N}\right]^{T}$ is defined by combining with the solutions of $\mathbf{S}_{i}$ to represent a solution of the overall formation control system $\mathbf{S}$ modeled in (11). Note that the choice of coefficients $k_{i},(i=1,2, \ldots, N)$ reflects that all vehicles in the formation could have completely different dynamics. Its time derivative $\dot{\mathbf{V}}$ is now given in

$$
\begin{aligned}
\dot{\mathbf{V}}= & k_{1} \dot{V}_{1}\left(\mathbf{x}_{1}\right)+k_{2} \dot{V}_{2}\left(\mathbf{x}_{2}\right)+k_{3} \dot{V}_{3}\left(\mathbf{x}_{3}\right)+\cdots+k_{N} \dot{V}_{N}\left(\mathbf{x}_{N}\right) \\
= & \sum_{i=1}^{N} k_{i}\left(\operatorname{grad} V_{i}\right)^{T} A_{i} \mathbf{x}_{i}+\left[0+\left(\operatorname{grad} V_{1}\right)^{T} k_{1} h_{12}\left(t, \mathbf{x}_{2}\right)\right] \\
& +\left[\left(\operatorname{grad} V_{2}\right)^{T} k_{2} h_{21}\left(t, \mathbf{x}_{1}\right)+\left(\operatorname{grad} V_{2}\right)^{T} k_{2} h_{23}\left(t, \mathbf{x}_{3}\right)\right] \\
& +\left[\left(\operatorname{grad} V_{3}\right)^{T} k_{3} h_{32}\left(t, \mathbf{x}_{2}\right)+\left(\operatorname{grad} V_{3}\right)^{T} k_{3} h_{34}\left(t, \mathbf{x}_{4}\right)\right] \\
& +\cdots+\left[\left(\operatorname{grad} V_{N-1}\right)^{T} k_{N-1} h_{(N-1) N}\left(t, \mathbf{x}_{N}\right)\right. \\
& \left.+\left(\operatorname{grad} V_{N}\right)^{T} k_{N} h_{N(N-1)}\left(t, \mathbf{x}_{N-1}\right)\right] .
\end{aligned}
$$

By taking the norm on the right-hand side of (21) and using the estimates in (16) and (19), $\dot{\mathbf{V}}$ is written as

$$
\begin{aligned}
\dot{\mathbf{V}} \leq & \sum_{i=1}^{N}\left(-c_{i 3} c_{i 2}^{-1} k_{i} V_{i}\right)+\nabla_{\max } k_{\max } \\
& \times\left[h_{12}\left(t, \mathbf{x}_{2}\right)+h_{21}\left(t, \mathbf{x}_{1}\right)\right. \\
& \quad+h_{23}\left(t, \mathbf{x}_{3}\right)+h_{32}\left(t, \mathbf{x}_{2}\right)+h_{34}\left(t, \mathbf{x}_{4}\right)+\cdots \\
& \left.\quad+h_{(N-1) N}\left(t, \mathbf{x}_{N}\right)+h_{N(N-1)}\left(t, \mathbf{x}_{N-1}\right)\right] \\
\leq- & c_{\min }\left(k_{1} V_{1}+k_{2} V_{2}+k_{3} V_{3}+\cdots+k_{N} V_{N}\right) \\
+ & \nabla_{\max } k_{\max }\left[h_{12}\left(t, \mathbf{x}_{2}\right)+h_{21}\left(t, \mathbf{x}_{1}\right)\right. \\
& \quad+h_{23}\left(t, \mathbf{x}_{3}\right)+h_{32}\left(t, \mathbf{x}_{2}\right)+\cdots \\
& \left.\quad+h_{(N-1) N}\left(t, \mathbf{x}_{N}\right)+h_{N(N-1)}\left(t, \mathbf{x}_{N-1}\right)\right]
\end{aligned}
$$

where $k_{\max }=\max \left\{k_{1}, k_{2}, \ldots, k_{N}\right\}, \nabla_{\max }=\max \{\|(\operatorname{grad}$ $\left.\left.V_{1}\right)^{T}\|,\|\left(\operatorname{grad} V_{2}\right)^{T}\|, \ldots,\|\left(\operatorname{grad} V_{N}\right)^{T} \|\right\}$ and $c_{\min }=\min$ $\left\{c_{13} c_{12}^{-1}, c_{23} c_{22}^{-1}, \ldots, c_{N 3} c_{N 2}^{-1}\right\}>0$.

Moreover, depending on the characteristics of the CMIP, the interaction terms, $h_{\mathrm{ij}}, i \neq j,(i, j=1,2, \ldots, N)$ in the model of (11) correspond to the torques of the CMIP system. The result achieved in (55) can be used to generate the following equation:

$$
\begin{aligned}
h_{12}\left(t, \mathbf{x}_{2}\right) & +h_{21}\left(t, \mathbf{x}_{1}\right)+h_{23}\left(t, \mathbf{x}_{3}\right)+h_{32}\left(t, \mathbf{x}_{2}\right)+\cdots \\
& +h_{(N-1) N}\left(t, \mathbf{x}_{N}\right)+h_{N(N-1)}\left(t, \mathbf{x}_{N-1}\right)=0 .
\end{aligned}
$$

If all the decomposed IASs can be stabilized, these terms satisfy the condition in (23). Incorporating (23) in (22), the following differential inequality is obtained:

$\dot{\mathbf{V}} \leq-c_{\min }\left[k_{1} V_{1}\left(\mathbf{x}_{1}\right)+k_{2} V_{2}\left(\mathbf{x}_{2}\right)+\cdots+k_{N} V_{N}\left(\mathbf{x}_{N}\right)\right]=-c_{\min } \mathbf{V}$

The above inequality is valid for all $t \geq t_{0}$, and its solution is given by (25)

$$
\mathbf{V} \leq \mathbf{V}_{0} \exp \left[-c_{\min }\left(t-t_{0}\right)\right], \quad t \geq t_{0}
$$

where $\mathbf{V}_{0}=k_{1} V_{10}+k_{2} V_{20}+\cdots+k_{N} V_{N 0}$ is the initial value of $\mathbf{V}(\mathbf{X})$ for the overall formation system, and $V_{10}, V_{20}, \ldots, V_{N 0}$ denote the initial values corresponding to the IASs, $\mathbf{S}_{i}, i=$ $1,2, \ldots, N$. Then, using the first inequality in (16) for the functions $V_{1}\left(\mathbf{x}_{1}\right), V_{2}\left(\mathbf{x}_{2}\right), \ldots, V_{N}\left(\mathbf{x}_{N}\right)$, as well as the definition (20), the following inequalities hold:

$$
\begin{aligned}
\mathbf{V} & \geq k_{1} c_{11}\left\|\mathbf{x}_{1}\right\|+k_{2} c_{21}\left\|\mathbf{x}_{2}\right\|+\cdots+k_{N} c_{N 1}\left\|\mathbf{x}_{N}\right\| \\
& \geq c_{m}\left(\left\|\mathbf{x}_{1}\right\|+\left\|\mathbf{x}_{2}\right\|+\cdots+\left\|\mathbf{x}_{N}\right\|\right) \\
\mathbf{V}_{0} & \leq k_{1} c_{12}\left\|\mathbf{x}_{10}\right\|+k_{2} c_{22}\left\|\mathbf{x}_{20}\right\|+\cdots+k_{N} c_{N 2}\left\|\mathbf{x}_{N 0}\right\| \\
& \leq c_{M}\left(\left\|\mathbf{x}_{10}\right\|+\left\|\mathbf{x}_{20}\right\|+\cdots+\left\|\mathbf{x}_{N 0}\right\|\right)
\end{aligned}
$$

where $c_{m}=\min \left\{k_{1} c_{11}, k_{2} c_{21}, \ldots, k_{N} c_{N 1}\right\}>0, c_{M}=\max$ $\left\{k_{1} c_{12}, k_{2} c_{22}, \ldots, k_{N} c_{N 2}\right\}>0$, and $\mathbf{x}_{i 0}=\mathbf{x}_{i}\left(t_{0}\right), i=1$, $2, \ldots, N$, denote the initial states of all the IASs. As for the previously defined solution $\mathbf{X}=\left[\mathbf{x}_{1}, \mathbf{x}_{2}, \ldots, \mathbf{x}_{N}\right]^{T}$ of the whole formation system $\mathbf{S}$ in (11), the relationship between their norms [34] is given by

$$
\|\mathbf{X}\| \leq\left\|\mathbf{x}_{1}\right\|+\left\|\mathbf{x}_{2}\right\|+\cdots+\left\|\mathbf{x}_{N}\right\| \leq \sqrt{2}\|\mathbf{X}\| .
$$

Combining (26) and (27) generates two inequalities

$$
\mathbf{V} \geq c_{m}\|\mathbf{X}\|, \quad \mathbf{V}_{0} \leq \sqrt{2} c_{M}\left\|\mathbf{X}_{\mathbf{0}}\right\|
$$

and applying the above inequalities to (25) produces the final result for showing the stability of the overall formation. Thus

$$
\begin{aligned}
& c_{m}\|\mathbf{X}\| \leq \mathbf{V} \leq \sqrt{2} c_{M}\left\|\mathbf{X}_{0}\right\| \exp \left[-c_{\min }\left(t-t_{0}\right)\right]_{\max } \\
& \Rightarrow\|\mathbf{X}\| \leq \frac{\sqrt{2} c_{M}}{c_{m}}\left\|\mathbf{X}_{0}\right\| \exp \left[-c_{\min }\left(t-t_{0}\right)\right]_{\max } \\
& \Rightarrow\|\mathbf{X}\| \leq C\left\|\mathbf{X}_{0}\right\| \exp \left[-c_{\min }\left(t-t_{0}\right)\right]
\end{aligned}
$$

where $t \geq t_{0}, C=\sqrt{2} c_{M} / c_{m}>0$, and $\mathbf{X}$ is the solution of the whole formation system, $\mathbf{S}$, for the initial conditions $\left(t_{0}, \mathbf{X}_{\mathbf{0}}\right)$. Based on the Formation Stability Definition 2, the inequality in (29) indicates that the solution of the overall formation system exponentially converges to the equilibrium point $\mathbf{X}_{e}=C\left\|\mathbf{X}_{0}\right\|$, which is associated with the neighborhood of the desired formation.

This is a unique and significant result with great potential for improved formation control. With this, the decentralized formation controller is subsequently designed to guarantee the closed-loop stability of each IAS, and consequently maintain the stability of the overall formation. In the following sections, the EDA strategy will be applied to a group of mobile robots and the corresponding decentralized formation controllers are implemented to guarantee the formation stability. Simulation studies are performed to illustrate the feasibility and to verify the stability of the overall formation.

\section{Decentralized Formation Controller Design}

\section{A. Modeling of IASs and Control Framework}

The vehicle's model discussed in Section II-C is chosen as the plant here. The description provided below mainly focuses on the vehicle dynamics in the $y$-direction. However, the strategy is also applicable to the movement in the $x$-direction. It is assumed that all the pendulums in the CMIP are uniform rods and that no viscous force exists at the joints, in which case $I=m l^{2} / 3$ and $B_{c}=0$. Denoting $J=4 m l^{2} / 3$ and $u=\ddot{p}_{i}$ as the cart acceleration, the individual IP model in (47) then becomes

$$
J \ddot{\theta}=-m l u \cdot \cos \theta+m g l \sin \theta+T_{i \kappa_{i-1}}+T_{i \kappa_{i}}
$$




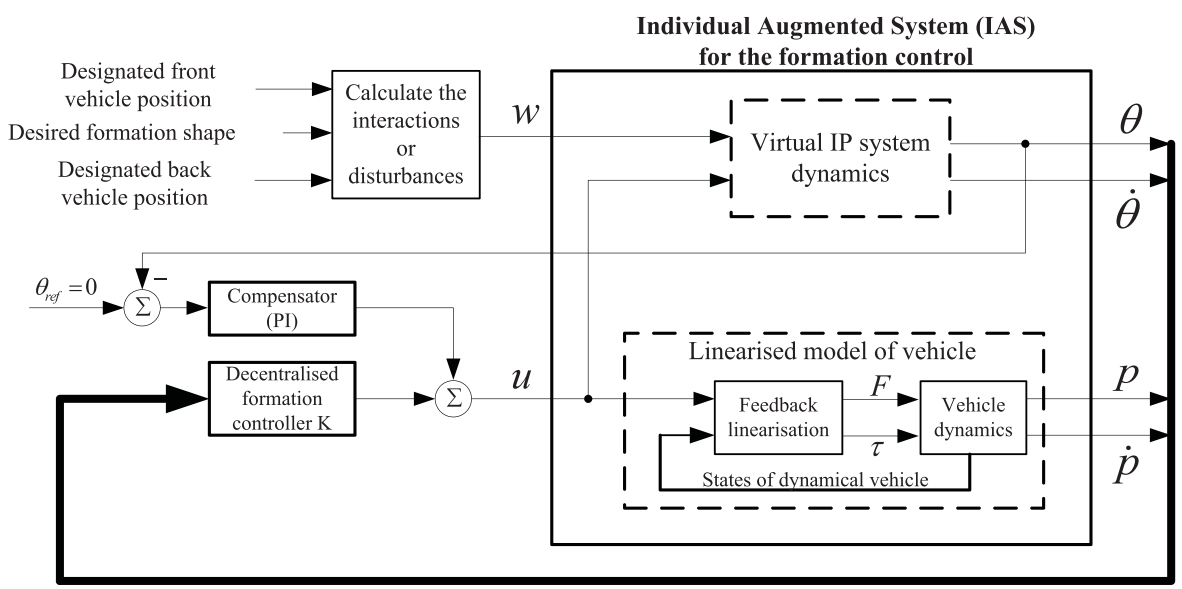

Fig. 6. Individual vehicle formation control framework.

and this equation can be linearized about the inverted position $(\theta=0 \mathrm{rad})$. If the change in $\theta$ always lies within a small neighborhood, (30) can then be rewritten as

$$
\ddot{\theta}=\frac{m g l}{J} \cdot \theta-\frac{m l}{J} \cdot u+\frac{w}{J}
$$

where $w$ is defined as the sum of $T_{i \kappa_{i-1}}$ and $T_{i \kappa_{i}}$. The above equation is combined with the linearized vehicle model to produce the IAS. Its differential equation is given by

$$
\left\{\begin{array}{l}
\ddot{p}=u_{c} \\
\ddot{\theta}=\frac{m g l}{J} \cdot \theta-\frac{m l}{J} \cdot u_{r}+\frac{1}{J} \cdot w
\end{array}\right.
$$

where $p$ is the position, $u_{c}$ is the commanded acceleration, and $u_{r}$ is the observed real-time acceleration of the vehicle. $u_{r}$ differs from $u_{c}$ physically, but their values are generally close, so it is feasible to consider them the same for simplicity and denote both of them by $u$. With the definition of (6), the local-formation vector is given as $\mathbf{x}_{F_{\mathrm{rd} \_} i}=[\theta, \dot{\theta}]^{T}$ and the state vector of each IAS is then expressed as $\mathbf{x}=\left[\tilde{\mathbf{x}}_{i}, \mathbf{x}_{F_{\text {rd } \_i}}\right]^{T}=$ $[p, \dot{p}, \theta, \dot{\theta}]^{T}$, which implies the augmentation of the state vector $\tilde{\mathbf{x}}$ for each vehicle. Thus, (32) becomes

$$
\frac{d}{d t}\left[\begin{array}{c}
p \\
\dot{p} \\
\theta \\
\dot{\theta}
\end{array}\right]=\underbrace{\left[\begin{array}{cccc}
0 & 1 & 0 & 0 \\
0 & 0 & 0 & 0 \\
0 & 0 & 0 & 1 \\
0 & 0 & \frac{m g l}{J} & 0
\end{array}\right]}_{A}\left[\begin{array}{c}
p \\
\dot{p} \\
\theta \\
\dot{\theta}
\end{array}\right]+\underbrace{\left[\begin{array}{c}
0 \\
1 \\
0 \\
\frac{-m l}{J}
\end{array}\right]}_{B_{2}} u+\underbrace{\left[\begin{array}{c}
0 \\
0 \\
0 \\
\frac{1}{J}
\end{array}\right]}_{B_{1}} w .
$$

The framework of an individual vehicle formation control is displayed in Fig. 6 on the basis of the EDA scheme, where the IAS is generated by the combination of the vehicle model and a virtual IP system. The exogenous input $w$ is derived from the formation change or error, and is considered here as a energy-bounded disturbance to the IAS. Subsequently, the LMI-based $H_{\infty}$ control methodology will be employed to reject this bounded disturbance and maintain the stability of each IAS.

Note that the decentralized $H_{\infty}$ controller is sufficient to maintain formation stability, but it generally cannot guarantee zero steady-state formation error that was defined in (5), because of the system type. This is the reason why the compensator in Fig. 6 is supplemented into the closed-loop system. Since the variation of $\theta$ is a reflection of the formation changing, the problem can be reduced into a standard regulation of the variable $\theta$. The PI-type compensator, $D(s)=$ $K_{p}\left(1+K_{i} / s\right)$, is thus employed for eliminating this steadystate formation error.

\section{B. LMI-Based $H_{\infty}$ Controller Design for IASs}

To stabilize the formation, the main design target of the decentralized formation controller is to reject the induced disturbances and guarantee the stability of the IASs. Here, the LMI-based $H_{\infty}$ control, which is well known for its disturbance rejection properties, is adopted. It is assumed that the state-space representation of each IAS is generalized into the multi-input/multioutput (MIMO) linear time-invariant system which is given by

$$
\begin{aligned}
\dot{\mathbf{x}} & =A \mathbf{x}+B_{1} \mathbf{w}+B_{2} \mathbf{u} \\
\mathbf{z} & =C_{1} \mathbf{x}+D_{11} \mathbf{w}+D_{12} \mathbf{u} \\
\mathbf{y} & =C_{2} \mathbf{x}+D_{21} \mathbf{w}+D_{22} \mathbf{u}
\end{aligned}
$$

where $\mathbf{x}$ is the state vector, $\mathbf{z}$ contains the outputs to analyze the performance of the control system, $\mathbf{y}$ is the measured output for the feedback, $\mathbf{u}$ is the control input, and $\mathbf{w}$ is the vector of exogenous input. A measure of $H_{\infty}$ performance is defined by (35), which is the gain $\left(\Gamma_{\mathrm{ee}}\right)$ from the exogenous input $\mathbf{w}$ to the output $\mathbf{z}$ of the IAS. To minimize the exogenous impact, i.e., to maximize the robust stability and performance of the closed-loop IAS, a state-feedback $H_{\infty}$ controller, $\mathbf{u}=K \mathbf{x}$ can be designed based on minimising $\Gamma_{\mathrm{ee}}$ under the bounded disturbance condition

$$
\Gamma_{\mathrm{ee}}=\frac{\|\mathbf{z}\|_{2}}{\|\mathbf{w}\|_{2}} \triangleq \frac{\int_{0}^{\infty}\left[\sum_{i=1}^{n} \lambda_{i}^{2} x_{i}^{2}(t)+\tau^{2} \mathbf{u}^{2}(t)\right] d t}{\int_{0}^{\infty} \mathbf{w}^{2}(t) d t}<\rho .
$$

In (35), $n$ is the state dimension, and $\lambda_{i}, \tau$ are the weighting factors for the state vector $\mathbf{x}$ and the input vector $\mathbf{u}$, respectively. Based on (35), the structure of the matrices $C_{1}$ and $D_{12}$ in (34) are given by

$$
C_{1}=\left[\begin{array}{c}
\operatorname{diag}\left(\lambda_{1}, \lambda_{2}, \ldots, \lambda_{n}\right) \\
0
\end{array}\right], \quad D_{12}=[\underbrace{0,0, \ldots, 0}_{n}, \tau]^{T}
$$




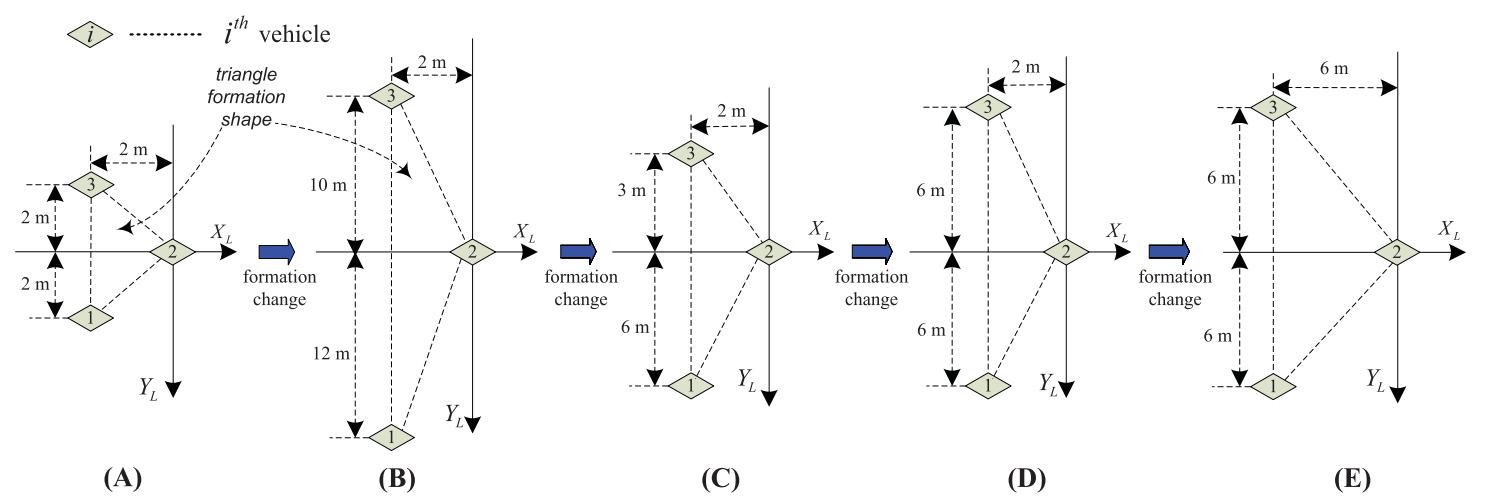

Fig. 7. Schematic of formation change process when maneuvering. (A)-(E) Represent different triangular formation shapes or geometries during maneuvering.

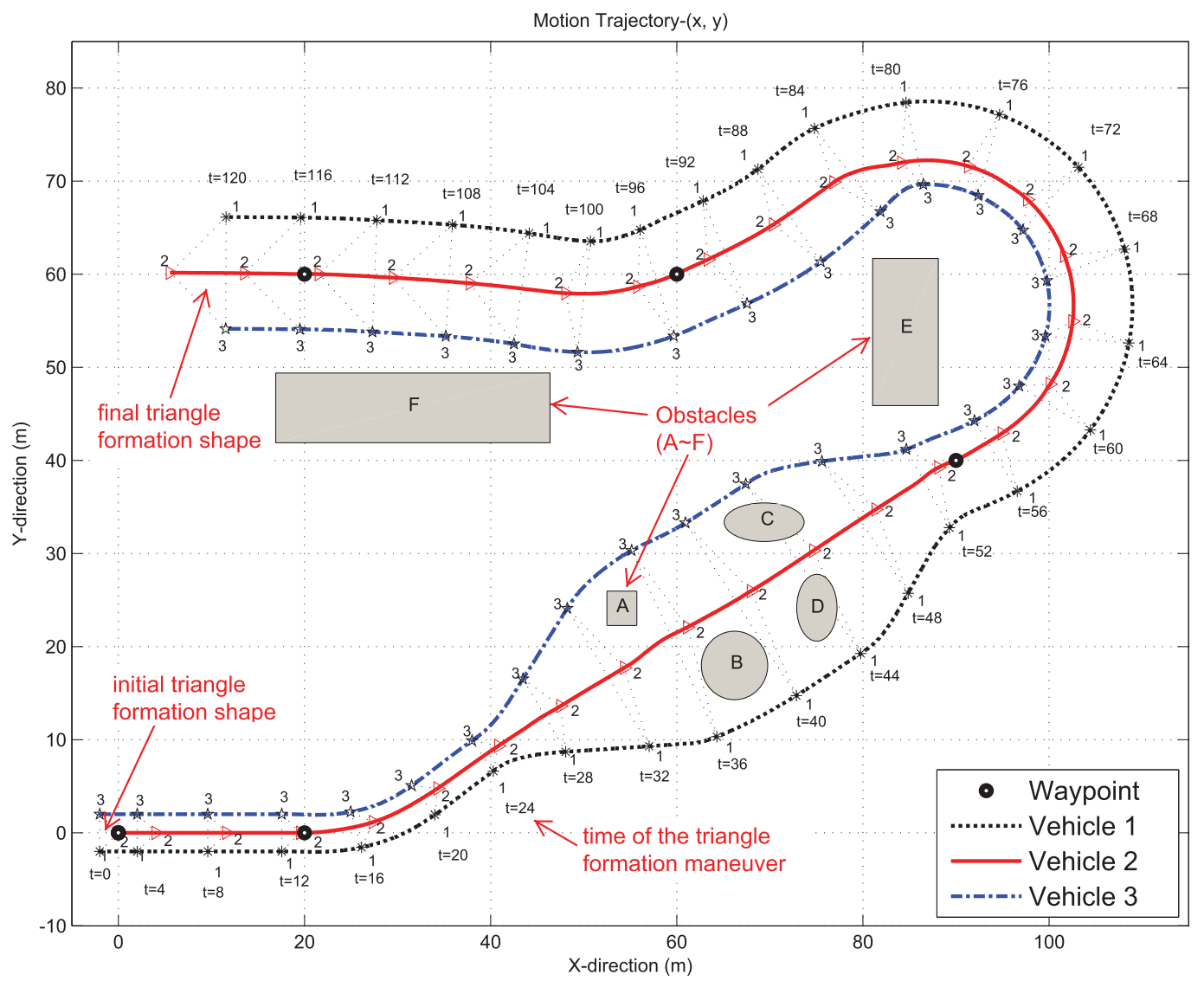

Fig. 8. Vehicles trajectories with formation maneuver corresponding to Fig. 7.

where "diag" denotes diagonal matrix, while the remaining matrices are determined from the plant itself.

For the stability of each IAS, the performance index, $\rho$ should be minimized to minimize the impact of the disturbance. The following design goal is to design the $H_{\infty}$ controller based on minimizing $\rho$, and to choose values of the weighting factors in (35) to make the system satisfy a predefined performance specification. Such an $H_{\infty}$ synthesis can be formulated as a convex optimization problem involving LMI [36], [37]. These LMIs correspond to the inequality counterparts of the usual Riccati equations for computing an $H_{\infty}$ controller. The state-feedback $H_{\infty}$ controller design can then be translated into the following optimization problem: minimize $\gamma$, such that the LMIs in (37) hold, where $\gamma=\rho^{2}$, if and only if there exist two symmetric positive definite matrices $X$ and $W$

$\left\{\begin{array}{ccc}A X+B_{2} W+\left(A X+B_{2} W\right)^{T} & B_{1} & \left(C_{1} X+D_{12} W\right)^{T} \\ B_{1}^{T} & -I & D_{11}^{T} \\ C_{1} X+D_{12} W & D_{11} & -\gamma I\end{array}\right]<0$.

This optimization problem can be solved using the LMI toolbox in MATLAB. Given the resultant optimal matrix variables $W$ and $X$, then $K=W X^{-1}$ is the gain matrix and $\mathbf{u}=$ $\left(W X^{-1}\right) \mathbf{x}$ is the required optimal state-feedback $H_{\infty}$ control input.

However, since the achieved $K$ is not constrained, the control input, $u$ is generally exceedingly larger than a practical 

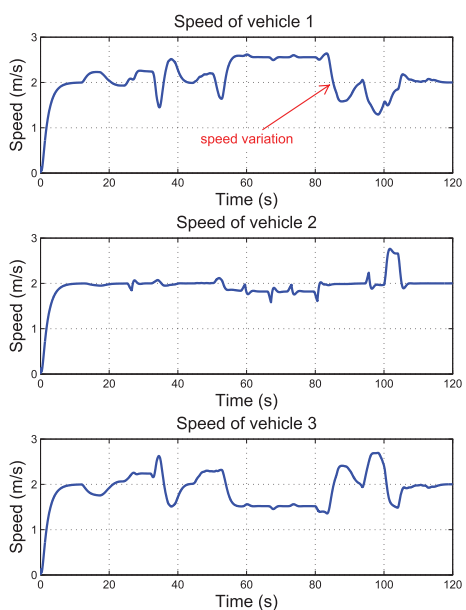

Fig. 9. Variation in speeds and orientations when maneuvering.
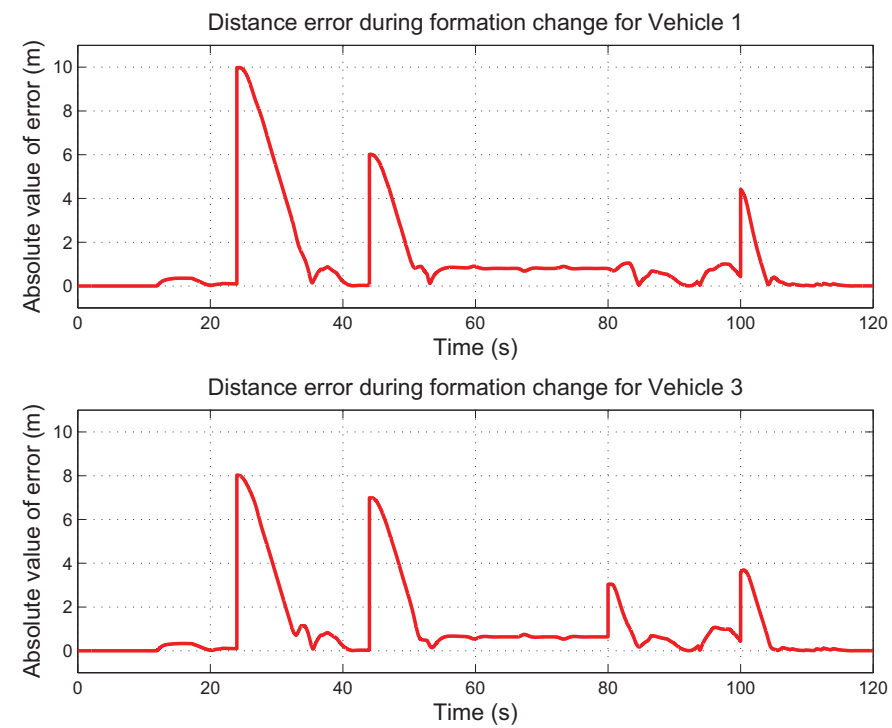

Fig. 10. Formation error of vehicles 1 and 3 when maneuvering.

actuator drive capability. Hence, it is necessary to limit its size by the inequality $K^{T} K<\alpha_{K} I$, where $\alpha_{K}>0$ and $I$ is the compatible identity matrix. Similarly, it is assumed that $X^{-1}<\alpha_{X} I$, where $\alpha_{X}>0$ [38]. By using the Schur complement formula, it can be expressed as an LMI

$$
\left[\begin{array}{cc}
\alpha_{X} & I \\
I & X
\end{array}\right]>0
$$

Thus

$$
\begin{aligned}
K^{T} K & =\left(W X^{-1}\right)^{T} W X^{-1} \\
& =X^{-1} W^{T} W X^{-1} \\
& <\left(\alpha_{X}\right)^{2} W^{T} W \\
& <\alpha_{K} I .
\end{aligned}
$$

It is obvious that there exists the inequality $W^{T} W<$ $\left(\alpha_{K} /\left(\alpha_{X}\right)^{2}\right) I=\alpha_{W} I$, where $\alpha_{W}=\alpha_{K} /\left(\alpha_{X}\right)^{2}$. This can also be represented as an LMI

$$
\left[\begin{array}{cc}
-\alpha_{W} I & W^{T} \\
W & -I
\end{array}\right]>0
$$
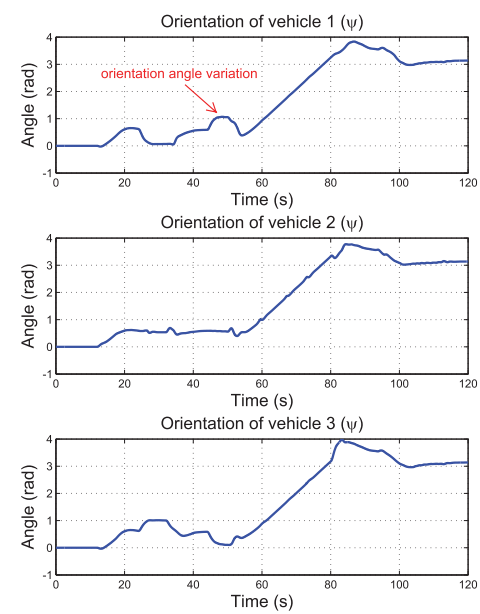

TABLE I

WAYPOINTS FOR THE VehicLES FoRMATION MANEUVER

\begin{tabular}{|c|c|c|c|c|c|}
\hline Waypoints No. & 1 & 2 & 3 & 4 & 5 \\
\hline$x(m)$ & 0 & 20 & 90 & 50 & 20 \\
\hline$y(m)$ & 0 & 0 & 40 & 60 & 60 \\
\hline
\end{tabular}

The above $\alpha_{X}$ and $\alpha_{W}$ constraints are now written as (41), where $C_{X}$ and $C_{W}$ can be selected by considering practical design specifications

$$
0<\alpha_{X}<C_{X} \quad 0<\alpha_{W}<C_{W} .
$$

Consequently, with above supplementaries, the previous optimization problem is restated as: minimize $\gamma$, such that the LMIs in (37), (38), (40), and (41) hold.

\section{IMPLEMENTATION AND SimUlations}

\section{A. Implementation of the Decentralized Formation Controller}

The state and output equations of the vehicle are given in (8) and (9), respectively. The parameter $L_{i}$ was chosen as $0.1 \mathrm{~m}$, and the linear and angular accelerations $\left[F_{i} / M_{i}, \tau_{i} / J_{\mathrm{vi}}\right]^{T}$ are designated as the vehicle inputs. The parameter values of the CMIP system are heuristically chosen as: $m=1 \mathrm{~kg}, g=$ $9.81 \mathrm{~m} / \mathrm{s}^{2}, l=h=0.3 \mathrm{~m}, J=(4 / 3) \mathrm{ml}^{2}=0.12 \mathrm{~kg} \cdot \mathrm{m}^{2}$, $k_{s}=1 \mathrm{~N} / \mathrm{s}$, but can be related to the dynamics of the physical system to be controlled. Based on these numerical values, the matrices of the model in (34) are given by

$$
A=\left[\begin{array}{cccc}
0 & 1 & 0 & 0 \\
0 & 0 & 0 & 0 \\
0 & 0 & 0 & 1 \\
0 & 0 & 24.525 & 0
\end{array}\right], \quad B_{1}=\left[\begin{array}{c}
0 \\
0 \\
0 \\
8.33
\end{array}\right], \quad B_{2}=\left[\begin{array}{c}
0 \\
1 \\
0 \\
-2.5
\end{array}\right] .
$$

The weighting factor values are heuristically chosen as: $\lambda_{1}=0.4, \lambda_{2}=0.1, \lambda_{3}=0.3, \lambda_{4}=0.3, \tau=0.3$, and 


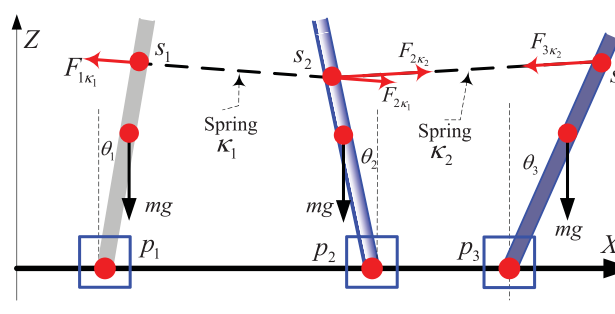

(A) Original structure

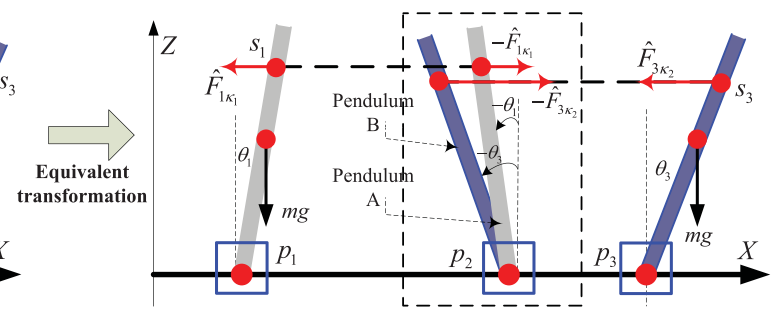

(B) Equivalent structure

Fig. 11. Equivalent transformation diagram. (A) Represents the original structure of the CMIP system with three inverted pendulums. (B) Equivalent structure corresponding to Fig. 11(A).

the other matrices in (34) then became

$$
\begin{aligned}
C_{1} & =\left[\begin{array}{c}
\operatorname{diag}(0.4,0.1,0.3,0.3) \\
0
\end{array}\right] \\
D_{11} & =[0,0,0,0,0]^{T} \\
D_{12} & =[0,0,0,0,0.3]^{T} \\
C_{2} & =\operatorname{diag}(1,1,1,1) \\
D_{21} & =[0,0,0,0]^{T} \\
D_{22} & =[0,0,0,0]^{T} .
\end{aligned}
$$

If the gain matrix $K$ is not constrained during the optimization, its value is given by (44) and $\min \{\gamma\}=1.9548$. Although the obtained optimal value for $\gamma$ seems reasonable, the value of $K$ is exceedingly large, and in turn gives an impractical control input

$$
K=10^{5} \times\left[\begin{array}{llll}
0.2236 & 0.3261 & 2.2925 & 0.4583
\end{array}\right]
$$

Once the constraints given by (38), (40), and (41) are imposed with $C_{X}=33, C_{W}=250$, the updated $K$ produced by LMI optimization is given by (45), and $\min \{\gamma\}=2.2532$

$$
K=\left[\begin{array}{llll}
21.3176 & 32.7525 & 234.5510 & 46.8746
\end{array}\right] .
$$

Furthermore, to eliminate the steady-state formation error, the parameters of the PI compensator are chosen as $K_{p}=10$, $K_{i}=15$ by trial-and-error procedure. These parameter values and control gains are used in all of the following simulations.

\section{B. Illustration of Formation Stability}

The group of vehicles was tasked to navigate the waypoints provided in Table I in addition to demanding a sequence of formation changes. Simulations are carried out to verify the stability of the forward and lateral formation during the desired formation change and navigation maneuvers. In this scenario, several initial configurations and definitions are given: 1) all the vehicles are initially stationary and positioned in the desired formation geometry; 2) the desired nominal forward speed of the formation group with respect to inertial reference frame was $2 \mathrm{~m} / \mathrm{s}$; and 3) the initial orientation angles $\psi_{i}$, $i=1,2,3$, of all vehicles are kept at $\psi_{i}=0 \mathrm{rad}$.

A line-of-sight (LOS) guidance strategy, which can be expressed by (46), was used to calculate the reference heading angle $\left(\psi_{\text {ref }}\right)$

$$
\psi_{\mathrm{ref}}=\int_{t_{0}}^{t} r_{h} d t, \quad r_{h}=\left\{\begin{array}{cc}
r_{\operatorname{maxT}}, & \text { if } \Delta \geq r_{\operatorname{maxT}} \\
r_{\mathrm{mid}}, & \text { if } r_{\mathrm{maxT}}>\Delta \geq r_{\mathrm{mid}} \\
r_{\mathrm{minT}}, & \text { if } r_{\operatorname{mid}}>\Delta \geq r_{\operatorname{minT}} \\
0, & \text { if } \Delta<r_{\operatorname{minT}}
\end{array}\right.
$$

where $r_{\operatorname{maxT}}$ and $r_{\operatorname{minT}}$ are the maximum and minimum heading rates for the physical vehicles, $\Delta$ is the angle difference from the current speed vector to the LOS, and $r_{\text {mid }}=r_{\operatorname{maxT}}+r_{\operatorname{minT}} / 2$. In the simulation, it is assumed that $r_{\max }=\pi / 27 \mathrm{rad}$, and $r_{\min T}=\pi / 108 \mathrm{rad}$, so $r_{\text {mid }} \approx 0.0727 \mathrm{rad}$.

Moreover, some obstacles are assumed to be present along the path and hence the formation changes are required to avoid them. Assuming that the positions of the obstacles labelled $\mathrm{A} \sim \mathrm{F}$ in Fig. 9 are known a priori, the reference formation shapes are obtained off-line and are shown in Fig. 7. Specifically, Fig. 7(A) shows the initial formation shape, Fig. 7(B) shows the first formation change at $t=24 \mathrm{~s}$ to avoid the obstacles (A, B, C, and D), Fig. 7(C) shows the formation contraction at $t=44 s$ to avoid the obstacle (E), Fig. 7 (D) shows the new lateral formation change at $t=80 \mathrm{~s}$, and finally Fig. 7 (E) shows the forward formation change at $t=100 \mathrm{~s}$.

The trajectories of the group of vehicles are displayed in Fig. 8 showing the turning maneuver and the formation change when needed, the associated speed and orientation being given in Fig. 9. In addition, the formation errors of the vehicles 1 and 3 relative to vehicle 2 are shown in Fig. 10. As shown in these figures, the following observations can be made.

1) The multivehicle formation remained stable during the turning maneuvers and successfully tracked all the given waypoints in addition to switching formation for avoiding collision. This observation further verifies the previous formation stability result.

2) Velocity variation with the formation turning or changing could be observed as it was required to maintain the positions of the vehicles within the new desired formation as quickly as possible. The speeds of the vehicles approached the reference speed $2 \mathrm{~m} / \mathrm{s}$ once the new formation shape was achieved.

3) During the turning maneuver and the formation changes, all the transient formation errors asymptotically converged to zero over time, while from $t=52 \mathrm{~s}$ to $t=80 \mathrm{~s}$ the small formation errors existed due to the desired cooperative turning motion.

Additional results can be found in [39] where the proposed algorithm has also been successfully applied to the model of an UAV. Extensive simulation results are provided demonstrating formation maintenance (disturbance rejection), formation change, and maneuvering in a 3-D space. 


\section{CONCLUSION}

In this paper, a complete methodology for solving the multivehicle formation control problem was developed. It employed the EDA strategy to support the design of the decentralized formation controller, and accordingly the associated formation stability was analyzed in detail. It was proven that with the support of the CMIP system, the stabilization of all the IASs was sufficient to guarantee the stability of the overall formation system. The LMI-based $H_{\infty}$ robust controllers were applied to reject the impact of the formation changes and maintain the stability of IASs, and subsequently guaranteed the overall formation stability. Simulation studies were performed to verify the achieved formation stability result. Future work includes the formation stability analysis under dynamic formation topologies, and the application in the formation flight of UAV in three dimensions.

\section{APPENDIX}

\section{Mathematical Modeling AND PROPERTy of CHAINED CMIP SYSTEM}

The chained CMIP system in Fig. 5 consists of $N$ cartmounted inverted pendulums (IP) coupled by $N-1$ springs. The points $s_{i-1}, s_{i}, s_{i+1}$ denote where the springs are attached to the pendulums, whereas $p_{i-1}, p_{i}, p_{i+1}$ are the positions of the $(i-1)$ th, $i$ th, $(i+1)$ th carts, respectively. Note that $F_{i \kappa_{i-1}}$ and $F_{i \kappa_{i}}$ denote the forces on the $i$ th pendulum exerted by the connected $\kappa_{i-1}$ and $\kappa_{i}$ springs, respectively. The model of the $i$ th inverted pendulum can be mathematically expressed by

$\left(I+m l^{2}\right) \ddot{\theta}_{i}+B_{c} \dot{\theta}_{i}=-m l \ddot{p}_{i} \cos \theta_{i}+m g l \sin \theta_{i}+T_{i \kappa_{i-1}}+T_{i \kappa_{i}}$

where $I=m l^{2} / 3$ is the moment of inertia for the pendulum, $B_{c}$ is the viscous damping constant at the pivot point, $\theta_{i}$ is the $i$ th deflection angle of pendulum from its vertical position (clockwise rotation is positive), $l$ and $h$ are the lengths from the pivot to the gravity center of the pendulum and to the joint point with the springs, respectively, $m$ and $g$ are the mass and the gravitational constant, respectively, and $T_{i \kappa_{i-1}}$ and $T_{i \kappa_{i}}$ are the torques on the $i$ th pendulum exerted by the associated forces $F_{i \kappa_{i-1}}$ and $F_{i \kappa_{i}}$. The complete model of the chained CMIP system with $N$ inverted pendulums is then given by

$$
\left\{\begin{array}{l}
\left(I+m l^{2}\right) \ddot{\theta}_{1}+B_{c} \dot{\theta}_{1}=-m l \ddot{p}_{1} \cos \theta_{1}+m g l \sin \theta_{1}+T_{1 \kappa_{1}} \\
\left(I+m l^{2}\right) \ddot{\theta}_{2}+B_{c} \dot{\theta}_{2}=-m l \ddot{p}_{2} \cos \theta_{2}+m g l \sin \theta_{2}+T_{2 \kappa_{1}}+T_{2 \kappa_{2}} \\
\vdots \\
\left(I+m l^{2}\right) \ddot{\theta}_{i}+B_{c} \dot{\theta}_{i}=-m l \ddot{p}_{i} \cos \theta_{i}+m g l \sin \theta_{i}+T_{i \kappa_{i-1}}+T_{i \kappa_{i}} \\
\vdots \\
\left(I+m l^{2}\right) \ddot{\theta}_{N}+B_{c} \dot{\theta}_{N}=-m l \ddot{p}_{N} \cos \theta_{N}+m g l \sin \theta_{N}+T_{N \kappa_{N-1}} .
\end{array}\right.
$$

In (48), if all the individual IP systems are stable and operate around the nominal conditions, there exist $\ddot{\theta}_{i}=0, \dot{\theta}_{i}=0$ and $\ddot{p}_{i}=0,(i=1,2, \ldots, N)$. The following result is then obtained by adding the two sides of (48):

$$
\begin{aligned}
& T_{1 \kappa_{1}}+T_{2 \kappa_{1}}+T_{2 \kappa_{2}}+\cdots+T_{i \kappa_{i-1}}+T_{i \kappa_{i}}+\cdots+T_{N \kappa_{N-1}} \\
& +m g l\left(\sin \theta_{1}+\sin \theta_{2}+\cdots \sin \theta_{N}\right)=0 .
\end{aligned}
$$

To simplify the mathematical analysis, it is assumed that there are only three inverted pendulums as shown in Fig. 11(A). In this case, the following equations given by (50) exist provided that all the decentralized IP systems are stable, i.e., the relevant pendulums maintain their torque balanced conditions

$$
\begin{aligned}
T_{1 \kappa_{1}} & =-m g l \sin \theta_{1} \\
T_{2 \kappa_{1}}+T_{2 \kappa_{2}} & =-m g l \sin \theta_{2} \\
T_{3 \kappa_{2}} & =-m g l \sin \theta_{3} .
\end{aligned}
$$

Fig. 11(B) shows another equivalent structure of Fig. 11(A) with the middle $\left(2^{\text {nd }}\right)$ inverted pendulum decomposed into two equivalent pendulums (Pendulum A and Pendulum B). To maintain the torque balance of the middle IP in Fig. 11(B), its resultant torque should satisfy

$$
\begin{aligned}
&-\hat{F}_{3 \kappa_{2}} h \cos \left(-\theta_{3}\right)+\left(-\hat{F}_{1 \kappa_{1}}\right) h \cos \left(-\theta_{1}\right) \\
&-m g l \sin \left(-\theta_{3}\right)-m g l \sin \left(-\theta_{1}\right)=0 .
\end{aligned}
$$

The sum of the first two terms on the left of (51) is the resultant torque exerted by the connected springs. Recalling the second equation in (50), and the following equation shown in (52) holds:

$$
\begin{aligned}
-\hat{F}_{3 \kappa_{2}} h \cos \left(-\theta_{3}\right)+ & \left(-\hat{F}_{1 \kappa_{1}}\right) h \cos \left(-\theta_{1}\right) \\
& =-\left(T_{2 \kappa_{1}}+T_{2 \kappa_{2}}\right)=m g l \sin \theta_{2} .
\end{aligned}
$$

Combining (51) and (52) generates the equation

$$
\sin \theta_{1}+\sin \theta_{2}+\sin \theta_{3}=0 .
$$

This result can be easily generalized to the CMIP with $\mathrm{N}$ pendulums, and the general result is given by

$$
\sin \theta_{1}+\sin \theta_{2}+\sin \theta_{3}+\cdots+\sin \theta_{N}=0 .
$$

Combining (49) and (54) yields

$$
T_{1 \kappa_{1}}+T_{2 \kappa_{1}}+T_{2 \kappa_{2}}+\cdots+T_{i \kappa_{i-1}}+T_{i \kappa_{i}}+\cdots+T_{N \kappa_{N-1}}=0 .
$$

Further, if the CMIP system is combined with the mesh topology, which is widely used in practical, the equation as similar as (54) can be expressed as

$$
\sum_{j=1, j \neq 1}^{N} T_{1 j}+\sum_{j=1, j \neq 2}^{N} T_{2 j}+\cdots+\sum_{j=1, j \neq N}^{N} T_{N j}=0
$$

where $T_{i j}$ is the torque on the $i$ th pendulum exerted by the interacted $j$ th pendulum. Similar result related to the tree topology can be also achieved by the same way.

In a word, the achieved equations above crucially show that if all the individual pendulums are stable, the resultant torque of the whole CMIP is equal to zero whatever the interconnection formation topology is. This useful result can be used to support the overall formation stability analysis of the multivehicle formation.

\section{REFERENCES}

[1] T. Arai, E. Pagello, and L. E. Parker, "Editorial: Advances in multirobot systems," IEEE Trans. Robot. Autom., vol. 18, no. 5, pp. 655-661, Oct. 2002.

[2] R. M. Murray, "Recent research in cooperative control of multi-vehicle systems,” J. Dynamic Syst., Meas., Control, vol. 129, no. 5, pp. 571-583, 2007. 
[3] T. Balch and R. Arkin, "Behavior-based formation control for multirobot teams," IEEE Trans. Robot. Autom., vol. 14, no. 6, pp. 926-939, Dec. 1998.

[4] J. R. T. Lawton, R. W. Beard, and B. J. Young, "A decentralized approach to formation maneuvers," IEEE Trans. Robot. Autom., vol. 19, no. 6, pp. 933-941, Dec. 2003.

[5] S. Monteiro and E. Bicho, "Attractor dynamics approach to formation control: Theory and application," Auto. Robot., vol. 29, nos. 3-4, pp. 331-355, 2010.

[6] J. P. Desai, J. P. Ostrowski, and V. Kumar, "Control of formations for multiple robots," in Proc. IEEE Int. Conf. Robot. Autom., May 1998, pp. 2864-2869.

[7] A. K. Das, R. Fierro, V. Kumar, J. P. Ostrowski, J. Spletzer, and C. J. Taylor, "A vision-based formation control framework," IEEE Trans. Robot. Autom., vol. 18, no. 5, pp. 813-825, Oct. 2002.

[8] W. Wang and J.-J. E. Slotine, "A theoretical study of different leader roles in networks," IEEE Trans. Autom. Control, vol. 51, no. 7, pp. 1156-1161, Jul. 2006.

[9] D. Gu and $\mathrm{H}$. Hu, "A model predictive controller for robots to follow a virtual leader," Robotica, vol. 27, pp. 905-913, Oct. 2009.

[10] M. Defoort, T. Floquet, A. Kokosy, and W. Perruquetti, "Sliding-mode formation control for cooperative autonomous mobile robots," IEEE Trans. Ind. Electron., vol. 55, no. 11, pp. 3944-3953, Nov. 2008.

[11] K. Tan and M. A. Lewis, "Virtual structures for high-precision cooperative mobile robotic control," Auto. Robot., vol. 4, no. 4, pp. 387-403, Oct. 1997.

[12] R. W. Beard and F. Y. Hadaegh, "Constellation templates: An approach to autonomous formation flying," in Proc. World Autom. Congr., 1998, pp. 1771-1776.

[13] I. F. Ihue, R. Skjetne, and T. I. Fossen, "Nonlinear formation control of marine craft with experimental results," in Proc. IEEE Conf. Decision Control, vol. 1. Dec. 2004, pp. 680-685.

[14] W. Ren and R. W. Beard, "Decentralized scheme for spacecraft formation flying via the virtual structure approach," J. Guid., Control, Dynamics, vol. 27, no. 1, pp. 73-82, 2004.

[15] D. H. Kim, H. Wang, and S. Shin, "Decentralized control of autonomous swarm systems using artificial potential functions: Analytical design guidelines," J. Intell. Robot. Syst., vol. 45, no. 4, pp. 369-394, 2006.

[16] T. Balch and M. Hybinette, "Social potentials for scalable multirobot formations," in Proc. Int. Conf. Robot. Autom., 2000, pp. 73-80.

[17] K. D. Do, "Bounded controllers for formation stabilization of mobile agents with limited sensing ranges," IEEE Trans. Autom. Control, vol. 52, no. 3, pp. 569-576, Mar. 2007.

[18] M. Arcak, "Passivity as a design tool for group coordination," IEEE Trans. Autom. Control, vol. 52, no. 8, pp. 1380-1390, Aug. 2007.

[19] D. M. Stipanovic, G. Inalhan, R. Teo, and C. J. Tomlin, "Decentralized overlapping control of a formation of unmanned aerial vehicles," Automatica, vol. 40, no. 8, pp. 1285-1296, 2004.

[20] S. Chung and J. Slotine, "Cooperative robot control and concurrent synchronization of lagrangian systems," IEEE Trans. Robot., vol. 25, no. 3, pp. 686-700, Dec. 2009.

[21] J. Corfmat and A. Morse, "Decentralized control of linear multivariate systems," Automatica, vol. 12, no, 5, pp. 476-495, 1976.

[22] J. A. Fax and R. M. Murray, "Information flow and cooperative control of vehicle formations," IEEE Trans. Autom. Control, vol. 49, no. 9, pp. 1465-1476, Sep. 2004.

[23] J. P. Desai, J. P. Ostrowski, and V. Kumar, "Modeling and control of formations of nonholonomic mobile robots," IEEE Trans. Robot. Autom., vol. 17, no. 6, pp. 905-908, Dec. 2001.

[24] P. Ögren, M. Egerstedt, and X. M. Hu, "A control Lyapunov function approach to multiagent coordination," IEEE Trans. Robot. Autom. vol. 18 , no. 5 , pp. $847-851$, Oct. 2002.

[25] D. Xue, J. Yao, G. Chen, and Y. L. Yu, "Formation control of networked multi-agent system," IET Control Theory Appl., vol. 4, no. 10, pp. 2168-2176, 2010.

[26] Y. Hong, L. Gao, D. Cheng, and J. Hu, "Lyapunov-based approach to multiagent systems with switching jointly connected interconnection," IEEE Trans. Autom. Control, vol. 52, no. 5, pp. 943-948, May 2007.

[27] J. T. Feddema, C. Lewis, and D. A. Schoenwald, "Decentralized control of cooperative robotic vehicles: Theory and application," IEEE Trans. Robot. Autom., vol. 18, no. 5, pp. 852-864, Oct. 2002.
[28] A. Yang, W. Naeem, G. W. Irwin, and K. Li, "Novel decentralised formation control for unmanned vehicles," in Proc. IEEE Intell. Veh. Symp., Jun. 2012, pp. 13-18.

[29] J. Shao, G. Xie, and L. Wang, "Leader-following formation control of multiple mobile vehicles," IET Control Theory Appl., vol. 1, no. 2, pp. 545-552, 2007.

[30] A. Yang, W. Naeem, G. W. Irwin, and K. Li, "A decentralised control strategy for formation flight of unmanned aerial vehicles," in Proc. Int. Conf. Control, Sep. 2012, pp. 345-350.

[31] D. D. Šiljak, Decentralized Control of Complex Systems. San Diego, CA, USA: Academic Press, 1991.

[32] A. Yang, G. W. Irwin, W. Naeem, and K. Li, "Application of wireless network control to a two inverted pendulum system," in Proc. IET Irish Syst. Signals Conf., Jun. 2011, pp. 1-6.

[33] J. Han, "From PID to active disturbance rejection control," IEEE Trans. Ind. Electron., vol. 56, no. 3, pp. 900-906, Mar. 2009.

[34] D. D. Šiljak, Large-Scale Dynamic Systems: Stability and Structure. The Netherlands, North Holland, Nov. 1978.

[35] H. K. Khalil, Nonlinear Systems, 3rd ed. Saddle River, NJ, USA: Prentice Hall, Dec. 2001.

[36] M. Chilali and P. Gahinet, " $\mathrm{H}_{\infty}$ design with pole placement constraints: An LMI approach," IEEE Trans. Autom. Control, vol. 41, no. 3, pp. 358-367, Mar. 1996.

[37] S. Boyd, L. E. Ghaoui, E. Feron, and V. Balakrishnan, Linear Matrix Inequalities in System and Control Theory (Studies in Applied Mathematics). Philadelphia, PA, USA: SIAM, Jun. 1994.

[38] D. D. Šiljak and D. M. Stipanović, "Robust stabilization of nonlinear systems: The LMI approach," Math. Problems Eng., vol. 6, no. 5, pp. $461-493,2000$

[39] A. Yang, "Novel decentralised formation control strategy for unmanned vehicles," Ph.D. dissertation, Dept. Electr. Eng. Comput. Sci., Queen's Univ. Belfast, Belfast, U.K., 2012

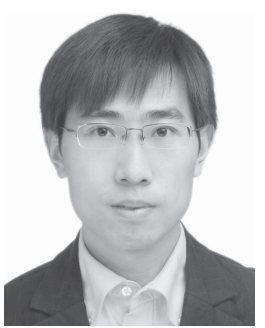

Aolei Yang received the B.Sc. degree from the Hubei University of Technology, Hubei, China, in 2004, the M.Sc. degree from Shanghai University, Shanghai, China, in 2009, and the Ph.D. degree from Queen's University Belfast, Belfast, U.K., in 2012.

$\mathrm{He}$ is currently with the School of Mechatronic Engineering and Automation, Shanghai University. His current research interests include wireless networked control systems, stability analysis of decentralized control framework, cooperative control of multiagents, and formation flight control of unmanned aerial vehicles.

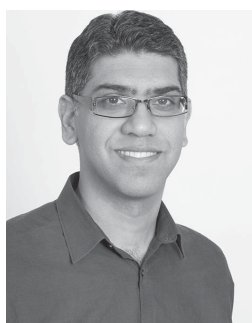

Wasif Naeem received the B.Eng. degree from NED University, Karachi, Pakistan, the M.Sc. degrees from King Fahd University, Dhahran, Saudi Arabia, in 1998 and 2001, respectively, both in electrical engineering, and the $\mathrm{Ph} . \mathrm{D}$. degree in mechanical and marine engineering from the University of Plymouth, Plymouth, U.K., in 2004.

$\mathrm{He}$ is currently a Lecturer with the School of Electronics, Electrical Engineering and Computer Science, Queen's University Belfast, Belfast, U.K. His current research interests include optimal and robust control, system identification, multivehicle formation control, and systems engineering. He has authored over 50 peer-reviewed journal and conference papers and two IET book chapters.

Dr. Naeem was a recipient of the Michael Richey Medal and the Denny Medal in 2008 and 2010 by the Royal Institute of Navigation and the Institution of Marine Engineering, Science and Technology, respectively. He is a member of the IFAC technical committee on marine systems and has served on the committee of a number of international conferences. 


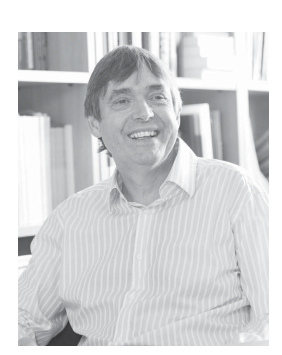

George W. Irwin (F'04) received the Honours degree in electrical and electronic engineering, the Ph.D. degree in control theory, and the D.Sc. degree from Queens University Belfast, Belfast, U.K., in 1972, 1976, and 1998, respectively.

$\mathrm{He}$ has been a personal Chair in Control Engineering since 1989. His research covering identification, monitoring, and control, including neural networks, fuzzy neural systems and multivariate statistics has led to some 350 research publications, including 125 peer-reviewed journal papers. Awards received include four IEE Premiums and two medals from the U.K. Institute of Measurement and Control.

Prof. Irwin has been elected fellow of the U.K. Royal Academy of Engineering and a member of the Royal Irish Academy. He is a Fellow of IFAC. He is a former Editor-in-Chief of Control Engineering Practice and he is currently serves on the Technical Board and the Publications Management Board of IFAC.

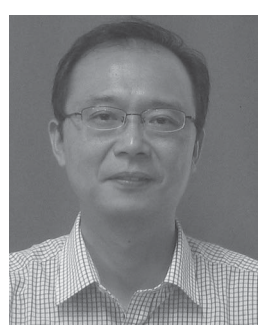

Kang Li (M'05-SM'11) received the B.Sc. degree from Xiangtan University, Hunan, China, in 1989, the M.Sc. degree from the Harbin Institute of Technology, Harbin, China, in 1992, and the Ph.D. degree from Shanghai Jiaotong University, Shanghai, China, in 1995 .

$\mathrm{He}$ is currently a Professor of intelligent systems and control with the School of Electronics, Electrical Engineering, and Computer Science, Queen's University Belfast, Belfast, U.K. He has published over 180 papers and edited 10 conference proceedings (Springer). His current research interests include nonlinear system modeling, identification, and control, bio-inspired computational intelligence, faultdiagnosis and detection, with recent applications on power systems and renewable energy, polymer extrusion processes, bioinformatics with applications on food safety, healthcare, and biomedical engineering. 